\title{
Simulating the superheating of nanomaterials due to latent heat release in surface reconstruction
}

\author{
Amit Singh, E. B. Tadmor* \\ Department of Aerospace Engineering and Mechanics, The University of Minnesota, Minneapolis, MN 55455
}

\begin{abstract}
Surface reconstruction and phase transformation in nanomaterials can result in a significant rise in temperature due to the release of stored energy called "latent heat." To simulate this behavior, we propose a hybrid continuum partial differential equation for non-Fourier heat transfer with a stochastic source term modeled using a kinetic Monte Carlo (KMC) algorithm for time-dependent rates to account for the latent heat release as the temperature is changing. The parameters required for the method are obtained through independent atomistic calculations. This includes energy barriers for KMC rates obtained using nudged elastic band calculations, and the non-Fourier thermal parameters obtained using a novel thermal parameter identification scheme described in this paper. As a demonstration of the approach, we study the superheating of silicon nanobeams observed in non-equilibrium molecular dynamics (NEMD) simulations. In these simulations, silicon (001) surfaces undergo a reconstruction from the ideal diamond crystalline surface to a reconstructed structure involving the formation of rows of dimers along a $\langle 110\rangle$ direction. This reconstruction is accompanied by latent heat release that causes the nanobeam to dramatically superheat. The evolution of the nonuniform temperature profile along the nanobeam predicted by the continuum-KMC method is in excellent agreement with the NEMD results. A logarithmic dependence of the superheating temperature on nanobeam length is observed and theoretically discussed.
\end{abstract}

Keywords: Surface reconstruction, Silicon, Molecular dynamics, Non-Fourier heat transfer, Cattaneo model, Hybrid continuum-KMC model, Latent heat modeling

\section{Introduction}

Surface reconstruction and phase transformations in materials are often accompanied by the release of stored energy referred to as "latent heat." In nanomaterials, where the surface-to-volume ratio is high, this energy constitute a significant increase to the overall energy of the system and can therefore cause significant heating. A recent example is described by Zheng et al [1] who observed superheating of $\mathrm{Cu}_{2} \mathrm{~S}$ nanorods due to phase transformations from a low-chalcocite to a high-chalcocite phase induced by electron beam heating. Our objective in this paper is to discuss the phenomenon of superheating due to latent heat release and to develop a predictive simulation methodology for problems of this nature using parameters obtained separately by atomistic calculations.

As a motivating example, we focus on the relatively simple problem, observed in non-equilibrium molecular dynamics (NEMD) simulations that we performed, of superheating of silicon nanobeams terminated by $\{001\}$ surfaces. The $\mathrm{Si}(001)$ surface is known to undergo a reconstruction that involves the formation of rows of dimers along $\langle 110\rangle$ directions [2]. In the NEMD simulation, the

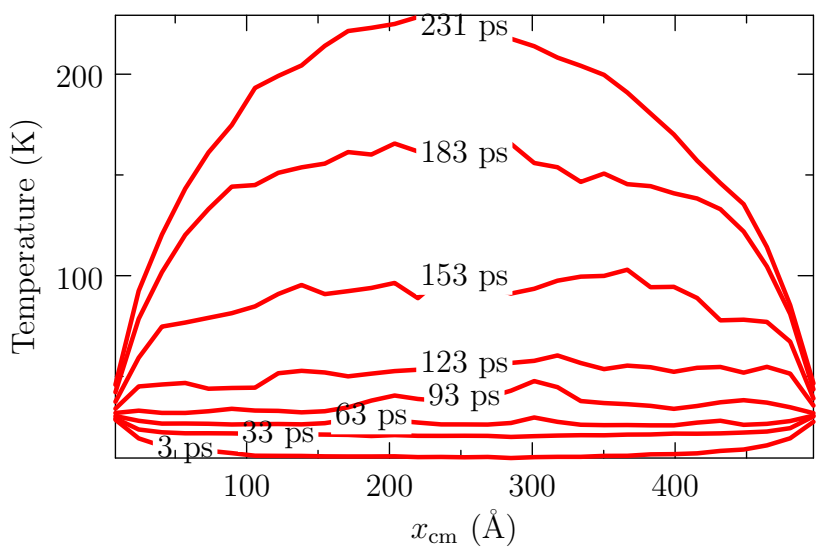

Figure 1: NEMD temperature profiles at increasing time for a silicon nanobeam of size $505 \times 16.3 \times 16.3 \AA$ with an initial temperature of $10 \mathrm{~K}$ and with the ends held at $30 \mathrm{~K}$. Each profile is an average over 3 ps. The profiles are marked with their corresponding times.

\footnotetext{
* Corresponding author

Email address: tadmor@umn.edu (E. B. Tadmor)
} 
nanobeam temperature is initially at $10 \mathrm{~K}$ and then the temperature of both ends is raised to $30 \mathrm{~K}$ and maintained at this level. ${ }^{1}$ Figure 1 shows a series of temperature profiles at increasing time. The interior of the beam gradually heats up, but rather than stabilizing at $30 \mathrm{~K}$ as expected, a dramatic overshooting in temperature that exceeds $200 \mathrm{~K}$ occurs due to release of latent heat that accompanies dimer formation in the reconstruction process. The maximum temperature is reached after 231 ps after which the temperature gradually decreases back to $30 \mathrm{~K}$ as the excess heat is absorbed at the ends. This is an interesting example that captures all of the main features of the superheating phenomenon in a well-studied system. We stress however that this is only an academic example ${ }^{2}$ since in experiments under vacuum conditions silicon surfaces reconstruct at temperatures as low as [3] $5 \mathrm{~K}$.

To model the superheating phenomenon we propose a continuum partial differential equation (PDE) for nonFourier heat transfer [4] parametrized using a novel thermal parameter identification (TPI) scheme based on an iteratively reweighted least squares (IRLS) regression method The injection of energy due to the latent heat released by dimerization is modeled using a stochastic Kinetic Monte Carlo (KMC) algorithm [6] that is coupled to the continuum PDE through the source term. A time-dependent KMC approach [7] is used since the temperature along the nanobeam, and hence the transition rates, are changing during the KMC process. The KMC rates are estimated using harmonic transition state theory (h-TST) with the activation barrier for dimer formation computed from the atomistic model using the climbing-image nudged elastic band (CI-NEB) method [8]. We find that the predictions of the hybrid continuum-KMC method for the silicon nanobeam example are in excellent agreement with the NEMD results. Using our model we are able to study larger systems than are readily accessible via NEMD. We find a logarithmic dependence of the superheating temperature on nanobeam length and provide a theoretical explanation for this observation.

The structure of the paper is as follows. In Section 2, we begin with a brief overview of Fourier and non-Fourier heat conduction models. In Section 3, we describe the TPI-IRLS procedure for extracting thermal parameters from the NEMD temperature profiles obtained prior to surface reconstruction. The results of applying TPI-IRLS to silicon are described in Section 4. In Section 5, the

\footnotetext{
${ }^{1}$ Initially, periodic boundary conditions are applied in all directions to create a bulk during the equilibration stage at the initial temperature of $\theta_{0}=10 \mathrm{~K}$. Periodicity is then removed along the vertical direction of the beam so that the top (001) and bottom (001) surfaces are exposed. Periodicity is maintained along the other directions during the simulation, so that the "nanobeam" is really an infinite nanoslab with finite thickness. For this reason the side atoms on the (010) and (010) surfaces do not reconstruct.

${ }^{2}$ Since the focus of the paper is on the simulation methodology, with the silicon nanobeam problem serving as an example, we do not pursue quantum corrections that would improve the accuracy of predictions at low temperatures.
}

surface reconstruction on the $\mathrm{Si}(001)$ surface and activation barriers for dimerization are studied. In Section 6 , we describe the hybrid continuum-KMC method that models the latent heat released during the surface reconstruction process and predicts the evolution of the temperature profiles. We conclude in Section 7 with a summary and suggestions for future work.

\section{Fourier and non-Fourier heat transfer models}

The simplest theory of heat conduction assumes Fourier's law. For a one-dimensional (1D) homogeneous body, this can be written as

$$
q=-k(\theta) \frac{\partial \theta}{\partial x}
$$

where $q=q(x, t)$ and $\theta=\theta(x, t)$ are the heat flux and temperature, respectively, at position $x$ in the current configuration at time $t$, and $k(\theta)$ is the temperature-dependent thermal conductivity.

The energy equation for a rigid solid body without heat sources is (see for example Section 10.2 in [9])

$$
\frac{\partial e}{\partial t}=\gamma \frac{\partial \theta}{\partial t}=-\frac{\partial q}{\partial x}
$$

where $e=e(x, t)$ is the internal energy per unit volume and $\gamma=\partial e / \partial \theta$ is the volumetric heat capacity. Substituting the expression for $q(x, t)$ in Eq. (1) into Eq. (2), we obtain Fourier's heat equation for a temperature-dependent thermal conductivity:

$$
\gamma \frac{\partial \theta}{\partial t}=k^{\prime}(\theta)\left(\frac{\partial \theta}{\partial x}\right)^{2}+k(\theta) \frac{\partial^{2} \theta}{\partial x^{2}}
$$

where $k^{\prime}=d k / d \theta$. This is a parabolic PDE that describes heat transfer as a diffusive process and assumes infinite speed of heat propagation.

In order to account for wavelike heat propagation at finite speed, Cattaneo $[10,11]$ and Vernotte $[12,13]$ introduced a relaxation time $\tau_{q}$ into the heat flux constitutive relation in Eq. (1). This leads to a non-Fourier model for heat flux:

$$
\tau_{q} \frac{\partial q}{\partial t}+q=-k(\theta) \frac{\partial \theta}{\partial x} .
$$

Taking the derivative of Eq. (4) with respect to $x$ and substituting in the energy equation in Eq. (2), yields the Cattaneo-Vernotte (CV) wave equation,

$$
\gamma \tau_{q} \frac{\partial^{2} \theta}{\partial t^{2}}+\gamma \frac{\partial \theta}{\partial t}=k^{\prime}(\theta)\left(\frac{\partial \theta}{\partial x}\right)^{2}+k(\theta) \frac{\partial^{2} \theta}{\partial x^{2}}
$$

An alternative non-Fourier model for heat transfer was proposed by Joseph and Preziosi [4] based on earlier work by Gurtin and Pipkin [14] and Nunziato [15]. They refer to their model as a "Jeffreys-type" heat flux constitutive 
relation due to an analogy with a stress model developed by Jeffreys [16]. The Jeffreys-type model has the form [17]:

$$
\tau_{q} \frac{\partial q}{\partial t}+q=-k(\theta) \frac{\partial \theta}{\partial x}-\tau_{\theta} k(\theta) \frac{\partial^{2} \theta}{\partial x \partial t},
$$

where $\tau_{\theta}$ is an additional relaxation time. Following the procedure outlined above leads to a Jeffreys-type equation for the temperature:

$$
\begin{aligned}
\gamma \tau_{q} \frac{\partial^{2} \theta}{\partial t^{2}}+\gamma \frac{\partial \theta}{\partial t} & =k^{\prime}(\theta)\left(\frac{\partial \theta}{\partial x}\right)^{2}+k(\theta) \frac{\partial^{2} \theta}{\partial x^{2}} \\
& +\tau_{\theta}\left[k^{\prime}(\theta) \frac{\partial \theta}{\partial x} \frac{\partial^{2} \theta}{\partial x \partial t}+k(\theta) \frac{\partial^{3} \theta}{\partial x^{2} \partial t}\right] .
\end{aligned}
$$

Assuming the following phenomenological relation for the thermal conductivity,

$$
k(\theta)=k_{0}\left(\theta / \theta_{0}\right)^{s},
$$

where $k_{0}$ is the thermal conductivity at temperature $\theta_{0}$, and $s$ is a real exponent, the Jeffreys-type equation can be written as

$$
\begin{array}{r}
\frac{\partial \theta}{\partial t}=-\tau_{q} \frac{\partial^{2} \theta}{\partial t^{2}}+\frac{k_{0}}{\gamma}\left[\left(\frac{\theta}{\theta_{0}}\right)^{s}\left(\frac{s}{\theta}\left(\frac{\partial \theta}{\partial x}\right)^{2}+\frac{\partial^{2} \theta}{\partial x^{2}}\right)\right] \\
+\frac{k_{0} \tau_{\theta}}{\gamma}\left[\left(\frac{\theta}{\theta_{0}}\right)^{s}\left(\frac{s}{\theta} \frac{\partial \theta}{\partial x} \frac{\partial^{2} \theta}{\partial x \partial t}+\frac{\partial^{3} \theta}{\partial x^{2} \partial t}\right)\right] .
\end{array}
$$

The CV wave equation and the Fourier equation follow from this equation as special cases with $\tau_{\theta}=0$ and $\tau_{q}=$ $\tau_{\theta}=0$, respectively.

\section{Obtaining thermal parameters using the TPI- IRLS method}

As a first step toward modeling the behavior in Fig. 1, we seek to obtain the thermal parameters appearing in the continuum models described in Section 2 by performing NEMD simulations of silicon nanobeams with free ideal (001) surfaces. We choose a slender nanobeam of length $L$ and square cross-section of side $W$ with $L \gg W$. Given the slender nanobeam geometry, we approximate the threedimensional (3D) heat transfer problem as 1D consistent with the derivation in Section 2.

In order to apply temperature boundary conditions (BCs) for computing thermal parameters, the nanobeam is divided into $N_{\mathrm{b}}$ bins of equal size as shown in Fig. 2(a) for $N_{\mathrm{b}}=11$. The bins centers are located at $x_{n}=\Delta_{x} / 2+$ $(n-1) \Delta_{x}\left(n=1, \ldots, N_{\mathrm{b}}\right)$, where $\Delta_{x}$ is the bin length along the $x$-direction. The instantaneous temperature in a given bin is computed as a spatial average over the appropriate phase function across all atoms within it. The thermodynamic temperature of a bin is obtained as a time average of the instantaneous bin temperature over a predefined time interval of $\Delta_{t}$ (see details in [17]). In order to improve the statistics, simulations can be repeated multiple times with different initial conditions drawn from a

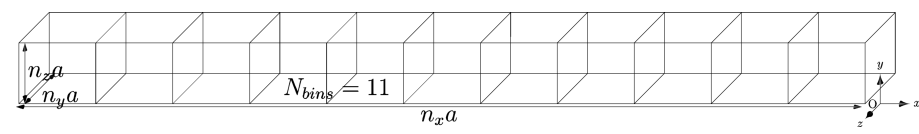

(a)

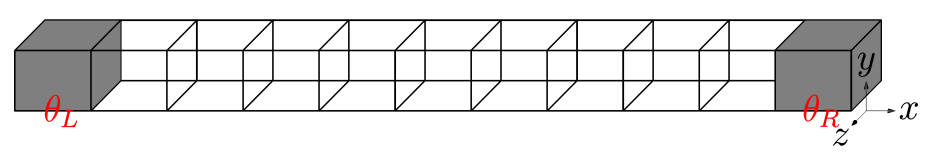

(b)

Figure 2: (a) Division of a nanobeam into bins in an NEMD simulation. (b) Thermal BCs applied to the beam for the TPI-IRLS method. Shaded regions are thermostated.

distribution associated with the initial temperature. Bin averages are then computed over all realizations of a given bin.

Initially, at time $t=0$, the nanobeam has a uniform temperature $\theta_{0}$ :

$$
\theta(x, 0)=\theta_{0}, \quad 0<x<L .
$$

Next the temperatures of the bins at the left and right ends of the nanobeam are set to new values, $\theta_{\mathrm{L}}$ and $\theta_{\mathrm{R}}$ :

$$
\theta(0, t)=\theta_{\mathrm{L}}, \quad \theta(L, t)=\theta_{\mathrm{R}} .
$$

In the NEMD simulations, the temperature BCs are applied to the bins at the ends of the nanobeam using a suitable thermostat as shown in Fig. 2(b).

In the TPI-IRLS approach, an NEMD simulation is performed starting with the initial condition in Eq. (11) and with the BCs specified in Eqs. (12). The resulting spatio-temporal temperature profiles after binning are then smoothed and a least squares regression method is used to determine the Jeffreys-type model parameters, $k_{0}, \tau_{q}$ and $\tau_{\theta}$, appearing in Eq. (10). (The same procedure can be applied to the Fourier or CV models to obtain their parameters.) ${ }^{3}$ We assume that the volumetric heat capacity $\gamma$ is constant over the temperature range of the simulation. We compute $\gamma$ from a separate molecular dynamics simulation at a temperature equal to the average of initial and $\mathrm{BC}$ temperatures.

\subsection{Temperature profile smoothing}

The binning procedure described above yields the temperature $\theta_{m n}$ of bin $n\left(n=1, \ldots, N_{\mathrm{b}}\right)$ at time $t_{m}=m \Delta_{t}$ ( $\left.m=0,1, \ldots, N_{\text {profiles }}\right)$, where $N_{\text {profiles }}$ is the number of

\footnotetext{
${ }^{3}$ Note that the TPI-IRLS approach differs from the TPI method we describe in [17], where an analytical solution for a decaying initial sinusoidal temperature profile is used to extract thermal parameters. A different approach is needed because the TPI method assumes that the thermal conductivity is independent of temperature, whereas in the present case the change in temperature is large and this assumption is not valid.
} 
stored temperature profiles. In the following, we refer to the initial $m=0$ profile as the "zeroth profile."

Although the bin temperatures $\theta_{m n}$ are obtained as an average over multiple NEMD simulations with different initial conditions, this data is still expected to be quite noisy due to the stochastic nature of NEMD and the small system size being studied. Therefore before attempting to fit this data to the continuum model in Eq. (10), it is necessary to apply a suitable smoothing procedure. We follow Müller and Timmer [18] and seek a surface $\tilde{\theta}(x, t)$ in $x-t$ space that "best" approximates the discrete bin data $\theta_{m n}$.

Different choices for the form of $\tilde{\theta}(x, t)$ can be made. Schoenberg [19] and Reinsch [20] suggested that a natural cubic spline was optimal for smoothing noisy data. However, splines have the propensity to follow the data too closely. At the other extreme, a least squares linear fit captures the average slope of the data without tracking deviations from the mean. We adopt a method described by de Boor [21] that uses a "smoothing parameter" $\alpha$ to balance between smoothness and closeness to the data. In this approach, $\alpha=0$ corresponds to a cubic spline and $\alpha=1$ corresponds to a linear least squares fit, with intermediate values corresponding to a mixture of the two. By tuning the value of $\alpha$ in the range $[0,1]$, the level of agreement with the data can be controlled. Since our data is discretized in both space $x$ and time $t$, smoothing is performed with a two-dimensional surface spline in the directions $x$ and $t$. The smoothing parameters in these directions are taken to be

$$
\alpha_{x}=e_{x} /\left(1+e_{x}\right), \quad \alpha_{t}=e_{t} /\left(1+e_{t}\right),
$$

where

$$
e_{x}=\left(\left(\Delta_{x}\right)^{3} / 16\right) \times 10^{a_{p}}, \quad e_{t}=\left(\left(\Delta_{t}\right)^{3} / 16\right) \times 10^{a_{p}} .
$$

The power $a_{p}$ can vary from 1 to 10 . A value of $a_{p}=$ 1 was used in most of our calculations. Data from the thermostated bins is excluded in the smoothing procedure.

\subsection{Iteratively reweighted least squares regression (IRLS)}

Next we turn to the least squares parameter extraction technique. The Jeffreys-type equation in Eq. (10) is assumed to hold at all points along the nanobeam at all time. It therefore holds at the bin centers $x_{n}\left(n=2, \ldots, N_{\mathrm{b}}-1\right)$ at times $t_{m}\left(m=1,2, \ldots, N_{\text {profiles }}\right)$. This provides a system of $N_{\text {data }}=\left(N_{\mathrm{b}}-2\right) \times N_{\text {profiles }}$ equations with which to determine the parameter vector $\boldsymbol{p}=\left(p_{1}, p_{2}, p_{3}\right)^{T}=$ $\left(\tau_{q}, k_{0} / \gamma, k_{0} \tau_{\theta} / \gamma\right)^{T}$. Formally, we write

$$
\left.\partial_{t} \theta\right|_{m n}=f\left(\boldsymbol{p},\left.\partial_{t t} \theta\right|_{m n},\left.\partial_{x x} \theta\right|_{m n},\left.\partial_{x x t} \theta\right|_{m n} ; s\right),
$$

where $\partial_{t} \theta \equiv \frac{\partial \theta}{\partial t}, \partial_{t t} \theta \equiv \frac{\partial^{2} \theta}{\partial t^{2}}, \partial_{x x} \theta \equiv\left(\frac{\theta}{\theta_{0}}\right)^{s}\left(\frac{s}{\theta}\left(\frac{\partial \theta}{\partial x}\right)^{2}+\right.$ $\left.\frac{\partial^{2} \theta}{\partial x^{2}}\right)$, and $\partial_{x x t} \theta \equiv\left(\frac{\theta}{\theta_{0}}\right)^{s}\left(\frac{s}{\theta} \frac{\partial \theta}{\partial x} \frac{\partial^{2} \theta}{\partial x \partial t}+\frac{\partial^{3} \theta}{\partial x^{2} \partial t}\right)$, for a given value of $s$ (the thermal conductivity exponent defined in
Eq. (9)). The notation $\left.(\cdot)\right|_{m n}$ means that the argument is evaluated at position $x_{n}$ at time $t_{m}$. So for example $\left.\partial_{t t} \theta\right|_{m n}=\left.\left(\partial^{2} \theta / \partial t^{2}\right)\right|_{\left(x_{n}, t_{m}\right)}$. Equation (14) is now approximated by replacing $\theta(x, t)$ with the function $\tilde{\theta}(x, t)$ obtained by the smoothing procedure described in the previous section:

$$
\left.\partial_{t} \tilde{\theta}\right|_{m n}=f\left(\boldsymbol{p},\left.\partial_{t t} \tilde{\theta}\right|_{m n},\left.\partial_{x x} \tilde{\theta}\right|_{m n},\left.\partial_{x x t} \tilde{\theta}\right|_{m n} ; s\right) .
$$

The derivatives are computed from the surface spline function.

From a regression standpoint, we take the left-hand side of Eq. (15), $y_{m n}=\left.\partial_{t} \tilde{\theta}\right|_{m n}$, to be the dependent vari$a b l e$, and the variables on the right, $\boldsymbol{X}_{m n}=\left(-\left.\partial_{t t} \tilde{\theta}\right|_{m n},\left.\partial_{x x} \tilde{\theta}\right|_{m n}, \partial_{x x}\right.$ to be the regressors (or "independent variables"). We now assume that the parameters $\boldsymbol{p}$ are constant over the range of temperatures being explored. We again emphasize that the regressors depend upon $s$, so that $\boldsymbol{p}$ is being estimated for a given value of $s$. We drop $s$ in the formal representation for the sake of clarity.

Examining Eq. (10), we see that a linear relationship exists between $y_{m n}$ and $\boldsymbol{X}_{m n}$,

$$
y_{m n}=\boldsymbol{X}_{m n} \cdot \boldsymbol{p}+R_{m n}
$$

where $R_{m n}$ are residual terms representing the noise in the data. The residuals are not known a priori and are obtained by inverting Eq. (16) for a given choice of $\boldsymbol{p}$,

$$
R_{m n}(\boldsymbol{p})=y_{m n}-\boldsymbol{X}_{m n} \cdot \boldsymbol{p} .
$$

The $N_{\text {data }}$ equations in Eq. (16) can be represented in compact form as

$$
\boldsymbol{y}=\boldsymbol{X} \boldsymbol{p}+\boldsymbol{R}
$$

where $\boldsymbol{y}=\left\{y_{m n}\right\}$ and $\boldsymbol{R}=\left\{R_{m n}\right\}$ are $N_{\text {data }} \times 1$ vectors and $\boldsymbol{X}=\left\{\boldsymbol{X}_{m n}\right\}$ is an $N_{\text {data }} \times 3$ array. This system of equations is overdetermined since there are only three unknowns (the parameters $p_{1}, p_{2}$ and $p_{3}$ ) whereas the number of equations is $N_{\text {data }} \gg 3$. A coefficient inversion problem [22] must therefore be solved by minimizing the sum of the square of the residuals

$$
\begin{aligned}
\Gamma(\boldsymbol{p}) & =\sum_{m, n}\left(R_{m n}(\boldsymbol{p})\right)^{2} \\
& =\sum_{m, n}\left(y_{m n}-\boldsymbol{X}_{m n} \cdot \boldsymbol{p}\right)^{2} \equiv\|\boldsymbol{y}-\boldsymbol{X} \boldsymbol{p}\|_{2}^{2} .
\end{aligned}
$$

This is known as the $l^{2}$-norm least squares regression method. A limitation of this approach is that it a priori assumes that the system is homoscedastic, i.e., that the random noise variables $R_{m n}$ all have the same variance. If this is not the case and the system is heteroscedastic, and the results obtained by minimizing $\Gamma(\boldsymbol{p})$ will be invalid. Instead, a method that corrects for heteroscedasticity by estimating the variance of the variables along with the parameters must be used. An example of such an approach is 
the IRLS (iteratively reweighted least squares) regression method. We follow the IRLS implementation of the hybrid $l^{2}-l^{1}$ norm adopted by Guitton and Verschuur [5], where the minimizing function is

$$
\Gamma_{W}(\boldsymbol{p})=\|\boldsymbol{W}(\boldsymbol{y}-\boldsymbol{X} \boldsymbol{p})\|_{2}^{2},
$$

and $\boldsymbol{W}$ is the following diagonal $N_{\text {data }} \times N_{\text {data }}$ weighting matrix:

$$
\boldsymbol{W}=\operatorname{diag}\left(\frac{1}{\left(1+R_{m n}^{2} / \epsilon^{2}\right)^{1 / 4}}\right) .
$$

Here $\epsilon$ is a scaling constant that we take to be

$$
\epsilon=\max \frac{|\boldsymbol{y}|}{100} .
$$

With this choice of $\boldsymbol{W}$, minimizing $\Gamma_{W}(\boldsymbol{p})$ is equivalent to minimizing [5]:

$$
\mathcal{Z}(\boldsymbol{p})=\sum_{m, n} z\left(R_{m n}(\boldsymbol{p})\right)=\sum_{m, n}\left(\left[1+R_{m n}(\boldsymbol{p}) / \epsilon\right]^{1 / 2}-1\right)
$$

where $R_{m n}(\boldsymbol{p})$ is given in Eq. (17). For a given residual $R_{m n}$, we have

$$
z\left(R_{m n}\right) \approx \begin{cases}\frac{1}{2}\left(R_{m n} / \epsilon\right)^{2} & \text { for small }\left|R_{m n}\right| / \epsilon \\ \left|R_{m n}\right| / \epsilon, & \text { for large }\left|R_{m n}\right| / \epsilon\end{cases}
$$

Hence, we obtain an $l^{2}$ treatment for small residuals and an $l^{1}$ treatment for large residuals with a smooth transition between the two determined by the parameter $\epsilon$.

The IRLS algorithm consists of iteratively recomputing the weights $\boldsymbol{W}$ and the parameters $\boldsymbol{p}$ until a convergence criterion is satisfied. The algorithm proceeds as follows. Given $\boldsymbol{p}^{(k)}$ (the parameters vector at iteration $k$ ) the residual at iteration $k$ follows from Eq. (17) and the corresponding weighting matrix $\boldsymbol{W}^{(k)}$ from Eq. (21). The values of the parameters at iteration $k+1$ are then obtained by solving the weighted linear least squares problem:

$$
\begin{aligned}
\boldsymbol{p}^{(k+1)} & =\underset{\boldsymbol{p}}{\arg \min }\left\|\boldsymbol{W}^{(k)}(\boldsymbol{y}-\boldsymbol{X} \boldsymbol{p})\right\|_{2}^{2} \\
& =\left(\boldsymbol{X}^{T} \boldsymbol{W}^{(k)} \boldsymbol{X}\right)^{-1} \boldsymbol{X}^{T} \boldsymbol{W}^{(k)} \boldsymbol{y},
\end{aligned}
$$

where the operator "arg min" returns the value of the argument $\boldsymbol{p}$ that minimizes the function. The algorithm is initialized by setting $\boldsymbol{W}^{(0)}$ to the identity matrix. This is equivalent to obtaining the first guess for the parameters, $\boldsymbol{p}^{(1)}$, through a standard least squares fitting procedure by minimizing $\Gamma(\boldsymbol{p})$ in Eq. (19). The iterations in Eq. (25) continue until the minimizer $\mathcal{Z}$ defined in Eq. (23) converges to a specified tolerance, i.e., $\left|\mathcal{Z}\left(\boldsymbol{p}^{(k+1)}\right)-\mathcal{Z}\left(\boldsymbol{p}^{(k)}\right)\right|<$ tol. In our case, the tolerance was set to tol $=10^{-10}$.

The IRLS method described above is used to obtain the parameters $\boldsymbol{p}$ for a given value of $s$. To obtain $s$, we minimize the $l^{2}$-norm of the residual,

$$
\min _{s} \sqrt{\Gamma(\boldsymbol{p} ; s)}=\min _{s}\|\boldsymbol{y}-\boldsymbol{X}(s) \boldsymbol{p}(s)\|_{2},
$$

and obtain $\boldsymbol{p}(\tilde{s})$, where $\tilde{s}$ is the minimizer of Eq. (26).

\section{Numerical results for the thermal parameters}

The boundary-value problem described at the start of Section 3 of a silicon nanobeam with thermal BCs is solved using NEMD. The nanobeam is modeled as a collection of $N$ atoms that interact via the three-body SW interatomic potential [23] with parameters for silicon archived in OpenKIM [24-26]. For details on NEMD simulations, see for example [27].

Temperature BCs were applied using either a NoséHoover chain thermostat [28-31] or a Langevin thermostat [32, 33] using a definition for temperature invariant with respect to rigid-body modes due to Jellinek and Li [34]. The parallel molecular dynamics code LAMMPS $[35,36]$ was used to integrate the equations of motion with modifications suggested by Singh and Tadmor [17].

All simulations begin with an initialization stage during which the system is equilibrated to the initial target temperature $\theta_{0}$ and allowed to expand to its equilibrium volume. The silicon nanobeam has a diamond structure and is oriented so that the $x, y$ and $z$ axes coincide with the crystallographic [100], [010] and [001] directions. A nanobeam of size $n_{x} a_{0} \times n_{y} a_{0} \times n_{z} a_{0}$ (with $n_{y}=n_{z}$ ) is constructed where $a_{0}=5.43 \AA$ is the zero-temperature equilibrium constant for argon. Periodic BCs are applied in all directions, so during the initial phase the "nanobeam" is actually a bulk system. The initial momenta of all the atoms are selected from a Maxwell-Boltzmann distribution for twice the target temperature $\left(2 \theta_{0}\right)$. This is done because approximately half of the initial kinetic energy is transformed to potential energy due to the equipartition of energy, and so the final temperature after equilibration will be about half the original value and hence close to $\theta_{0}$. After assigning the initial velocities, the nanobeam is evolved subject to NPT conditions with the pressure set to $P=0$ and the temperature to $T=\theta_{0}$. A coupled NoséHoover chain thermostat (chain length $=3$ ) and barostat as described in Shinoda et al. [37] were used. The method is implemented within the LAMMPS code. The system is evolved for a total of $2 \times 10^{6} \mathrm{MD}$ steps with a time step of $\Delta t_{\mathrm{MD}}=2$ fs. Following the NPT simulation, the periodicity along $z$ axis is removed, and (001) and (001) surfaces are exposed. An NVT simulation follows for $2 \times 10^{6} \mathrm{MD}$ steps with the same time step in which only temperature is controlled to complete the initialization process. This is necessary to allow the surfaces to relax to their equilibrium structure at the target temperature.

We illustrate the TPI-IRLS method for a beam of size $33 \times 3 \times 3$ unit cells after an initialization stage with $\theta_{0} \approx$ $10 \mathrm{~K}$. The number of bins is set to $N_{\mathrm{b}}=11$ each containing 216 atoms. Next the temperature BCs in Eq. (12) are enforced by setting the temperatures of the leftmost and rightmost bins to $\theta_{\mathrm{L}}=\theta_{\mathrm{R}}=30 \mathrm{~K}$ using a Langevin thermostat with a damping time of $0.5 \mathrm{ps}$. The system is run for $2 \times 10^{5}$ MD steps with a time step of $\Delta t_{\mathrm{MD}}=1 \mathrm{fs}$. Thermal fluctuations are reduced by running the simulation ten times with different initial momenta drawn from 
a Maxwell-Boltzmann distribution for $\theta_{\mathrm{L}}=\theta_{\mathrm{R}}=30 \mathrm{~K}$. $N_{\text {profiles }}=200$ temperature profiles are stored by taking averages of instantaneous values over $\Delta_{t}=1$ ps. This temporal averaging ensures the existence of local thermodynamic equilibrium conditions [17]. Since we are interested in extracting the thermal parameters prior to the initiation of surface reconstruction, we only take the first 15 profiles (i.e. we only take temperature data from the first 15 ps of the simulation before any reconstructions are observed to occur). These profiles are spline smoothed according to the procedure outlined in Section 3.1, where the parameters $a_{p}, \Delta_{t}$ and $\Delta_{x}$ in Eq. (13) are equal to 1,1 ps and $16.29 \AA$, respectively.

We apply the IRLS method described in Section 3.2 and obtain the estimated coefficients $\boldsymbol{p}$ with components $p_{1}=\tau_{q}, p_{2}=k_{0} / \gamma$, and $p_{3}=k_{0} \tau_{\theta} / \gamma$. Only positive values of these coefficients are considered. Using the estimated parameters, the continuum Jeffreys-type model in Eq. (10) is numerically solved by employing the finite difference discretization scheme described in the online supplementary information (SI). We calculate the root-mean-square deviation (RMSD) in temperature values between the continuum model and the atomistic data. We obtain different $\boldsymbol{p}$ values for different subsets of profiles such as $m \in[0,3]$, $m \in[1,3]$, etc. (where $m$ is defined in Section 3.1) and select the $\boldsymbol{p}$ values for which the RMSD value is minimum.

The volumetric heat capacity $\gamma$ appearing in $p_{2}$ and $p_{3}$ is computed using a separate calculation. The entire beam is brought to steady state at $\theta=20 \mathrm{~K}$ with a Langevin thermostat for $2 \times 10^{6} \mathrm{MD}$ steps with a time step size $\Delta t_{\mathrm{MD}}=2 \mathrm{fs}$. The beam continues to be thermostated for another $3 \times 10^{5} \mathrm{MD}$ steps and the energy of the beam is recorded at each MD step. Assuming that the ergodic hypothesis holds, $\gamma$ is related to the average energy fluctuations through [38]

$$
k_{\mathrm{B}} \theta^{2} \gamma V=\left\langle E^{2}\right\rangle-\langle E\rangle^{2},
$$

where $k_{\mathrm{B}}$ is Boltzmann's constant. Using Eq. (27) we find $\gamma=1.40 \pm 0.10 \times 10^{-5} \mathrm{eV} /\left(\AA^{3} \mathrm{~K}\right)$. Substituting $\gamma$ into $p_{2}$ and $p_{3}$, the following results are obtained for the Jeffreys parameters for silicon, $s=-1.5 \pm 0.1, k_{0}=$ $16.36 \pm 1.25 \mathrm{~W} / \mathrm{mK}, \tau_{q}=5.18 \pm 0.36 \mathrm{ps}, \tau_{\theta}=1.50 \pm 0.45 \mathrm{ps}$. The error bars on the parameters are obtained using a statistical $t$-test procedure and all coefficients are estimated at a $5 \%$ statistical significance level [39]. The $t$-test provides the standard errors $\mathrm{SE}_{\mathrm{p}_{1}}, \mathrm{SE}_{\mathrm{p}_{2}}, \mathrm{SE}_{\mathrm{p}_{3}}$, for the three components of the parameter vector $\boldsymbol{p}$. The standard errors of $k_{0}, \tau_{q}, \tau_{\theta}$, i.e., $\mathrm{SE}_{\mathrm{k} 0}, \mathrm{SE}_{\tau_{\mathrm{q}}}, \mathrm{SE}_{\tau_{\theta}}$, are computed from $\mathrm{SE}_{\mathrm{p}_{1}}, \mathrm{SE}_{\mathrm{p}_{2}}, \mathrm{SE}_{\mathrm{p}_{3}}, \gamma$ and its standard deviation $\sigma_{\gamma}$ using the following relations:

$$
\begin{aligned}
\mathrm{SE}_{\tau_{\mathrm{q}}} & =\mathrm{SE}_{\mathrm{p}_{1}}, \\
\frac{\mathrm{SE}_{\mathrm{k}_{0}}^{2}}{k_{0}^{2}} & =\frac{\mathrm{SE}_{\mathrm{p}_{2}}^{2}}{p_{2}^{2}}+\frac{\sigma_{\gamma}^{2}}{\gamma^{2}}, \\
\frac{\mathrm{SE}_{\tau_{\theta}}^{2}}{\tau_{\theta}^{2}} & =\frac{\mathrm{SE}_{\mathrm{p}_{3}}^{2}}{p_{3}^{2}}+\frac{\mathrm{SE}_{\mathrm{p}_{2}}^{2}}{p_{2}^{2}},
\end{aligned}
$$

Table 1: The CV parameters for three different nanoslab sizes along $x$-axis in unit cell. The size along the $y$ and $z$ axis is 3 unit cells. Values in parentheses are the standard errors of the parameters.

\begin{tabular}{cccc}
\hline \hline size & $k_{0}[\mathrm{~W} / \mathrm{mK}]$ & $\tau_{q}[\mathrm{ps}]$ & $s$ \\
33 & $24.85(1.63)$ & $2.42(0.20)$ & $-1.9(0.1)$ \\
63 & $33.38(1.43)$ & $4.29(0.33)$ & $-1.9(0.1)$ \\
93 & $36.15(1.34)$ & $3.59(0.68)$ & $-1.9(0.1)$ \\
\hline \hline
\end{tabular}

which are derived based on the assumption that the covariance terms between $p_{2}, p_{3}$ and $\gamma$ are zero.

The TPI-IRLS procedure was also applied to the CV model (Eq. (5)) and the Fourier model (Eq. (3)) for the same size beam. We obtain $s=-1.9 \pm 0.1, k_{0}=24.85 \pm$ $1.63 \mathrm{~W} / \mathrm{mK}, \tau_{q}=2.42 \pm 0.20 \mathrm{ps}$ for the $\mathrm{CV}$ model and $s=-1.9 \pm 0.1, k_{0}=28.43 \pm 1.64 \mathrm{~W} / \mathrm{mK}$, for the Fourier model. Figure 3 shows a comparison between the NEMD temperature profiles and the continuum profiles for the Fourier, CV and Jeffreys-type models.

Close examination of Fig. 3 and statistical testing reveal that the CV and the Jeffreys-type models are in better agreement with the NEMD data. In particular, the RMSD in temperature for the Fourier model is 2.41 as opposed to 2.03 for the Jeffreys-type model and 1.57 for the CV model. For systems with different sizes we obtain similar results. Thus we find that the CV model provides the best fit for the atomistic data for silicon nanobeams. We therefore choose the CV model for further analysis. ${ }^{4}$ Results for the $\mathrm{CV}$ model for three different nanobeam sizes needed for the continuum-KMC calculations in Section 6.3 are given in Table 1. (Note that for nanoscale systems, thermal parameters are size-dependent due to phonon scattering effects when the phonon mean free path is comparable to the system characteristic size. For more on this, see for example [17, 40-43].)

In order to check the validity of the fitting scheme, we test whether the parameters obtained for one set of BCs can be used to describe a problem with different BCs. Figure 4 shows a comparison between atomistic NEMD data and the CV model for a different boundary-value problem with $\theta_{\mathrm{R}}=30 \mathrm{~K}$ and $\theta_{\mathrm{L}}=\theta_{0}=10 \mathrm{~K}$ using the parameters obtained from the problem in Fig. 3(b). The agreement is quite good. This suggests that CV provides a good model for thermal transport in silicon nanobeams and is transferable to different loading conditions.

\section{Surface Reconstruction}

In the fitting process described in Section 4, temperature profiles from only the first 15 ps were used (see Fig. 3

\footnotetext{
${ }^{4}$ We also note that Jeffreys-type parameters obtained using the TPI-IRLS procedure are not unique, whereas they are for the Fourier and CV models. Unique Jeffreys-type parameters can be obtained by including heat flux profiles in the fitting procedure [17]. This is another reason why we have chosen CV over the Jeffreys-type model for the present work.
} 


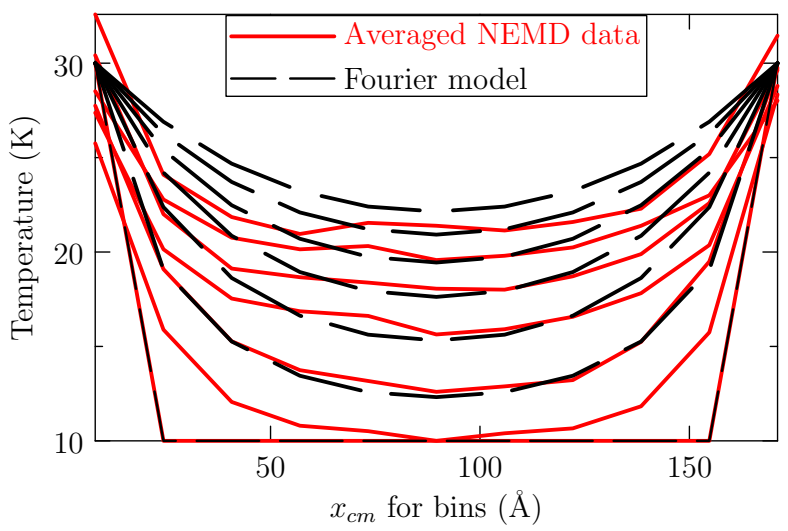

(a)

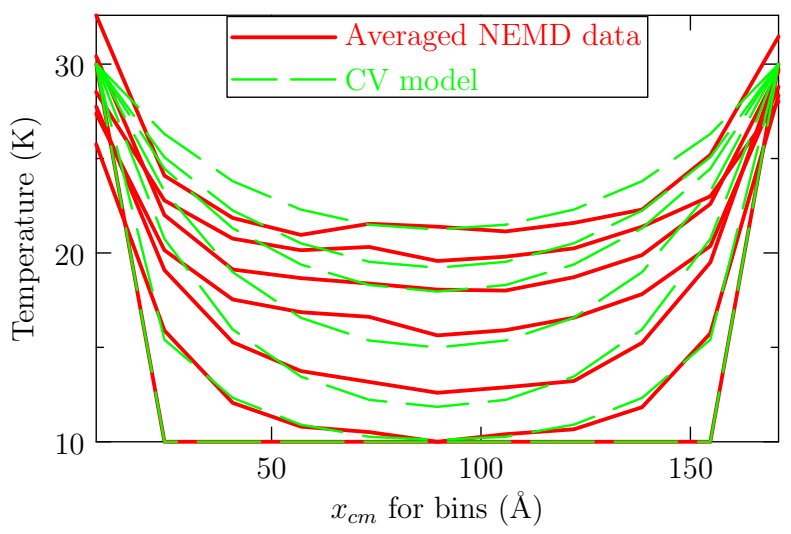

(b)

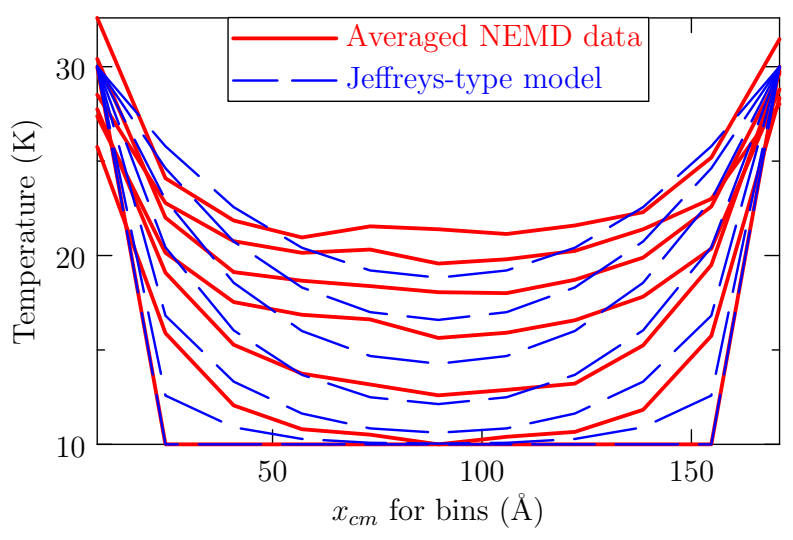

(c)

Figure 3: Comparison of the smoothed NEMD temperature profiles and the continuum models for (a) Fourier, (b) CV, and (c) Jeffreystype thermal models for a silicon nanobeam of size $33 \times 3 \times 3$ unit cells prior to surface reconstruction. The initial beam temperature is $\theta_{0}=10 \mathrm{~K}$ and the boundary temperatures are set to $30 \mathrm{~K}$. Profiles are separated by 6 ps and each profile corresponds to an average of $1 \mathrm{ps}$ of data. The zeroth profile is also shown.

and accompanying discussion). This is because at later times the surfaces of the nanobeam begin to reconstruct as the temperature rises. In this section we explore the energetics of the reconstruction process of an exposed (001) surface in a vacuum.

As noted in the introduction, the $\mathrm{Si}(001)$ surface is

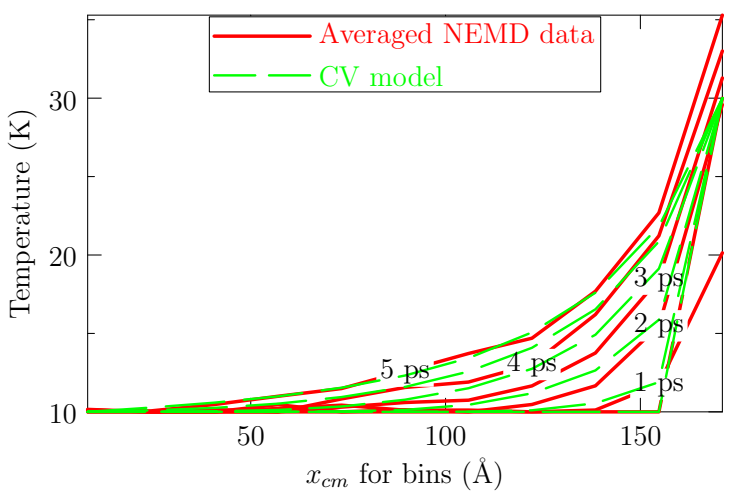

Figure 4: Comparison of the NEMD temperature profiles with the predictions of the continuum $\mathrm{CV}$ model using the thermal parameters obtained from the temperature profiles in Fig. 3(b). The initial beam temperature is $\theta_{0}=10 \mathrm{~K}$ and the temperature of the rightmost bin is increased to $30 \mathrm{~K}$. In all simulations, NEMD profiles are an average of ten runs with different initial momenta. Except for the zeroth profile, each profile is an average of 1 ps of data. The profiles are marked with their corresponding times.

known to undergo a simple reconstruction that involves the formation of rows of dimers along $\langle 110\rangle$ directions [2]. Historically, Schlier and Farnsworth [44] using low-energy electron diffraction (LEED) measurements first discovered the $p(2 \times 1)$ structure, which involves $2 \times 1$ periodic cells of the ideal surface structure. Later LEED experiments detected a $c(4 \times 2)$ pattern $[45,46]$. Scanning tunneling microscopy (STM) studies of the $\mathrm{Si}(001)$ surface at room temperature show both symmetric and asymmetric $p(2 \times 1)$ reconstructions [47]. The ground state configuration of the $\mathrm{Si}(001)$ surface at low temperature is still an issue of debate with arguments made for symmetric $p(2 \times 1)$ [48], asymmetric $p(2 \times 1)$ [3], $c(4 \times 2)$ [49], and a mixture of $c(4 \times 2), p(2 \times 2)$ and asymmetric $p(2 \times 1)$ [50-52]. Several authors [53-57] have studied the energetics of silicon surface reconstruction and provide estimates for the latent heat released per dimer from the ideal unreconstructed structure to the symmetric $p(2 \times 1)$, asymmetric $p(2 \times 1)$, $c(4 \times 2)$ and other reconstructions.

We start with an ideal unreconstructed (001) surface as shown in Fig. 5(a). The rows of dangling bonds, which are clearly visible in the figure, are less energetically favorable than the reconstructed states described below. For the SW potential, we find that dangling bonds on the surface rotate to form dimers along the [110] direction as shown in Fig. 5(b) (3D view) and Fig. 5(c) (plan view). This reconstruction is different from the experimentally observed structures discussed in Section 1. Rather than the commonly observed $p(2 \times 1)$ reconstruction, the SW potential predicts a structure consisting of rows of dimers along the [110] direction separated by a row of dangling bonds. We refer to this as the symmetric $p(3 \times 1)$ reconstruction. For the SW potential, the symmetric $p(2 \times 1)$ and symmetric $p(3 \times 1)$ structures have surface energies of $246.4 \mathrm{meV} / \AA^{2}$ and $259.3 \mathrm{meV} / \AA^{2}$, respectively. This means that the $p(2 \times 1)$ structure is more favorable than the $p(3 \times 1)$. 
However, as we show below, a small energy barrier exists for the formation of dimers. For SW, this barrier is a little higher for creating an adjacent dimer in the $p(2 \times 1)$ structure $(22.5 \mathrm{meV})$ than for creating a dimer separated by a row of dangling bonds in the $p(3 \times 1)$ structure $(20.8 \mathrm{meV})$. This difference is the reason that the SW potential favors the $p(3 \times 1)$ structure at low temperature. At higher temperatures, the system has more energy to overcome energy barriers and can more fully explore its configuration space. Due to entropic effects, a mixture of $p(2 \times 1)$ and $p(3 \times 1)$ structures is observed, with the fraction of $p(2 \times 1)$ increasing with temperature. This is clear in Figs. 5(d) and 5(e) that show the reconstructed surfaces predicted by SW at at $300 \mathrm{~K}$ and $1100 \mathrm{~K}$. The high temperature results are in agreement with other molecular dynamics simulations using the SW potential [58, 59].

The fact that dimerization is not observed at very low temperatures (over the duration of an NEMD simulation) but does occur at elevated temperature, suggests that it is an activated process. The h-TST transition rate $R$ for dimer formation is

$$
R=\nu_{d} \exp \left(-\Delta E_{b} / k_{\mathrm{B}} \theta\right)
$$

where $\nu_{d}$ is the attempt frequency, $\theta$ is the local temperature, $k_{\mathrm{B}}$ is Boltzmann's constant, and $\Delta E_{b}$ is the energy barrier separating the undimerized and dimerized states.

We use the CI-NEB method of Henkelman et al. [8] to obtain the energy barrier $\Delta E_{b}$ and also compute the energy released during dimerization $\Delta E_{r}$. We start with a nanobeam of size $33 \times 3 \times 3$ unit cells equilibrated at $10 \mathrm{~K}$ as discussed in Section 4 and focus on a single bin along the $[\overline{1} 10]$ direction. Taking the ideal unreconstructed (001) surface as the first configuration $\mathrm{C} 1$, we displace the two atoms in the front towards each other by $0.13 \AA$ and minimize the total energy to obtain the second configuration $\mathrm{C} 2$ with one dimer formed. We repeat the process for the next two atoms in the row to obtain the third configuration C3 with two dimers formed, and so on to obtain configurations $\mathrm{C} 4$ and $\mathrm{C} 5$ as shown in Fig. 6. Our intention in creating this sequence of configurations is to see whether the barrier and energy release for dimer formation is affected by previously formed dimers in the vicinity. We apply the CI-NEB procedure with $\mathrm{C} 1$ as the initial state and $\mathrm{C} 2$ as the final state with a total of 16 replicas along the band generated by linear interpolation. We repeat the process for $\mathrm{C} 2$ and $\mathrm{C} 3$, and so on. Figure 6(f) presents the energy along the minimum energy path for the resulting series of transitions. We find that the energy barrier $\Delta E_{b}$ for the first dimer formation is approximately $22 \mathrm{meV}$ and the energy released during this process is $\Delta E_{r} \approx 1.65 \mathrm{eV}$. The energy barrier and energy released in all subsequent dimerizations is about the same with $\Delta E_{b} \approx 6.8 \mathrm{meV}$ and $\Delta E_{r} \approx 1.71 \mathrm{eV}$. It is noteworthy that the barrier for the first dimerization is about three times higher than for subsequent dimerizations. Thus it is easier to dimerize after the process begins.

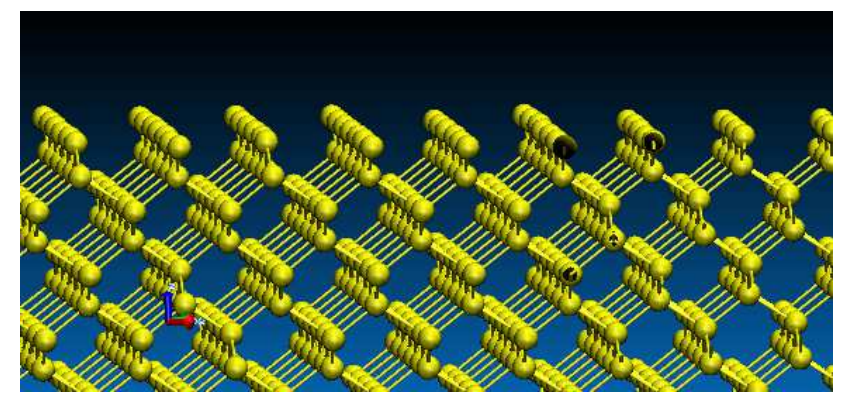

(a)

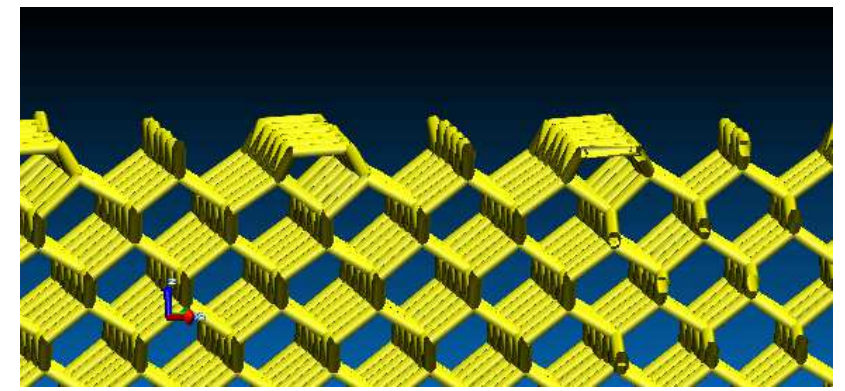

(b)

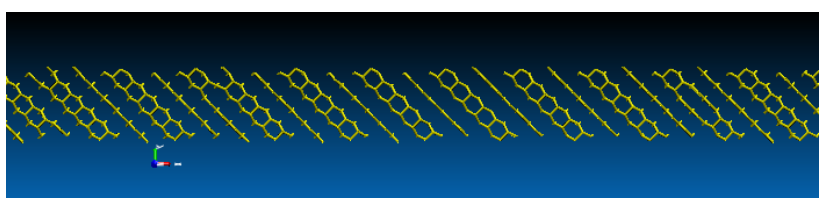

(c)

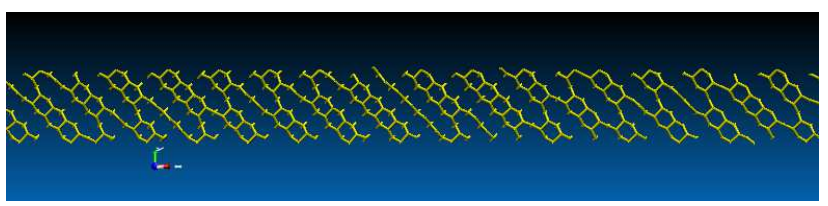

(d)

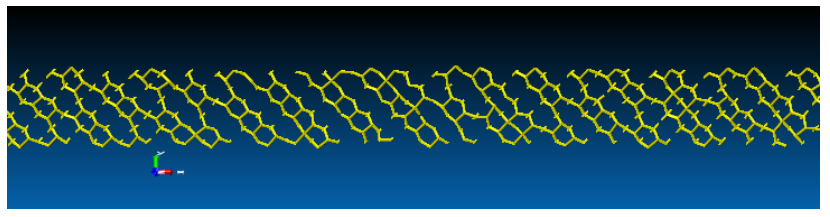

(e)

Figure 5: (a) 3D view of the ideal unreconstructed (001) surface, and (b) the reconstructed surface at $30 \mathrm{~K}$ of the silicon nanobeam of size $33 \times 3 \times 3$ unit cells. The top layer of the reconstructed (001) surface seen from above at (c) $30 \mathrm{~K}$, (d) $300 \mathrm{~K}$, (e) $1100 \mathrm{~K}$.

Instead of the process described above where all dimers form in a single row, it is also possible for dimers to form in alternate rows. A corresponding series of configurations $\mathrm{C} 1-\mathrm{C} 2-\mathrm{C} 3^{\prime}-\mathrm{C} 4^{\prime}-\mathrm{C} 5^{\prime}$ for this alternate process are shown in Fig. 7 (configurations $\mathrm{C} 1$ and $\mathrm{C} 2$ are the same ones shown in Figs. 6(a) and 6(b)). The energy along the corresponding minimum energy path obtained by CI-NEB is shown in Fig. 7(d). Three types of transitions are observed in this process:

Type I The first dimer formed on an ideal surface (C1- 


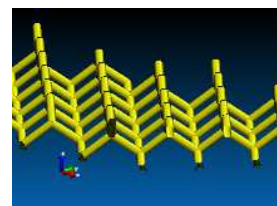

(a) $\mathrm{C} 1$

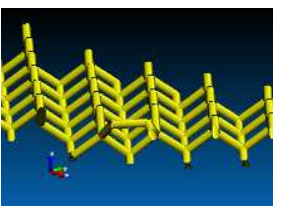

(b) $\mathrm{C} 2$

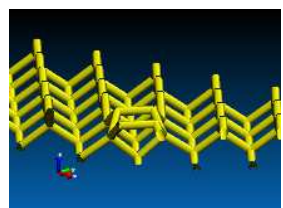

(c) $\mathrm{C} 3$

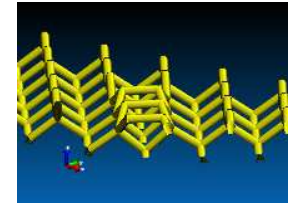

(d) $\mathrm{C} 4$

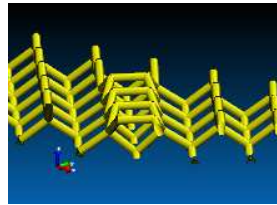

(e) $\mathrm{C} 5$

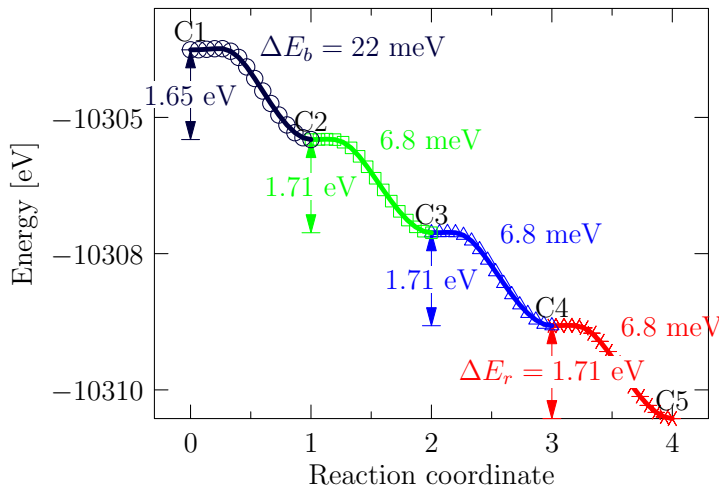

(f)

Figure 6: (a) Configuration $\mathrm{C} 1$ associated with the unreconstructed (001) surface, (b)-(e) configurations C2, C3, C4 and C5 with one, two, three and four dimers, respectively, and (f) the minimum energy path for the series of transitions from $\mathrm{C} 1$ to $\mathrm{C} 5$ indicated by different colors. The number on the left of each curve is the energy released $\Delta E_{r}$ and the number on the right is the energy barrier $\Delta E_{b}$.

C2) has $\Delta E_{b}=22 \mathrm{meV}$ and $\Delta E_{r}=1.65 \mathrm{eV}$ (as also reported in Fig. 6).

Type II A dimer formed on an alternate row to an adjacent one $\left(\mathrm{C} 2-\mathrm{C} 3^{\prime}\right.$ and $\left.\mathrm{C} 3^{\prime}-\mathrm{C} 4{ }^{\prime}\right)$ has $\Delta E_{b}=33 \mathrm{meV}$ and $\Delta E_{r}=1.61 \mathrm{eV}$.

Type III A dimer formed on the same row as an adjacent dimer $\left(\mathrm{C} 4^{\prime}-\mathrm{C} 5^{\prime}\right)$ has $\Delta E_{b}=6.8 \mathrm{meV}$ and $\Delta E_{r}=$ $1.71 \mathrm{eV}$ (as also reported in Fig. 6(f)).

These results indicate that dimer formation is easiest along the same row. The energy barrier for alternate dimerization is more than four times higher. In addition to the above, CI-NEB calculations were carried out for several other systems with free (001) surfaces for different system sizes $(3 \times 3 \times 4,6 \times 6 \times 4,33 \times 4 \times 4$, and others $)$ and different dimer configurations. In all cases, only Type I, II and III transitions were observed.

We calculated the attempt frequency $\nu_{d}$ appearing in

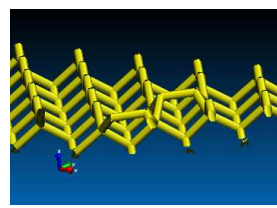

(a) $\mathrm{C} 3^{\prime}$

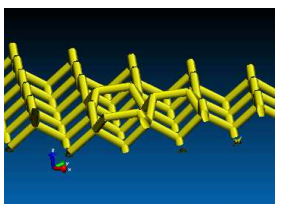

(b) $\mathrm{C}^{\prime}$

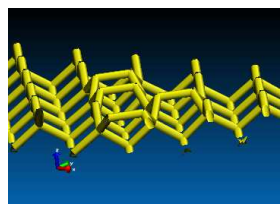

(c) $\mathrm{C}^{\prime}$

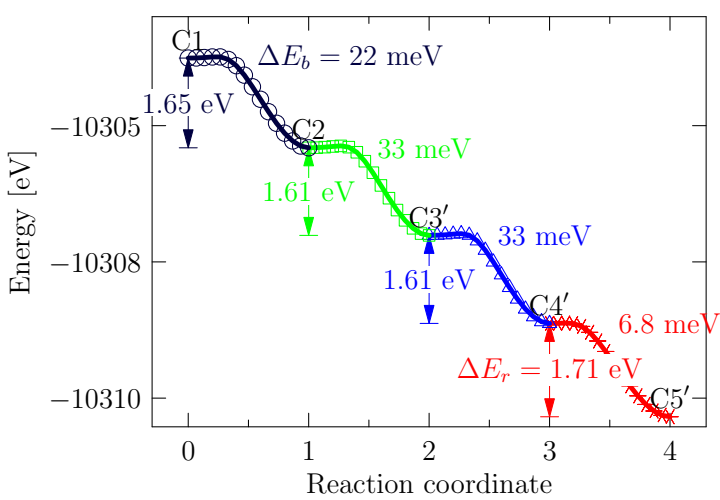

(d)

Figure 7: (a)-(c) show the configurations $\mathrm{C} 3^{\prime}, \mathrm{C} 4^{\prime}$, and $\mathrm{C}^{\prime}{ }^{\prime}$ with two, three and four dimers along two adjacent rows, and (d) The minimum energy path for $\mathrm{C} 1-\mathrm{C} 5^{\prime}$ transition. The black, green, blue and red curves are the minimum energy paths for $\mathrm{C} 1-\mathrm{C} 2, \mathrm{C} 2-\mathrm{C} 3^{\prime}$, $\mathrm{C} 3^{\prime}-\mathrm{C} 4^{\prime}$ and $\mathrm{C}^{\prime}-\mathrm{C}^{\prime}$ transitions. The numbers to the left of each curve is the energy released $\Delta E_{r}$ and the number to the right is the energy barrier $\Delta E_{b}$.

Eq. (29) using the Vineyard equation [60]:

$$
\nu_{d}=\frac{\prod_{j=1}^{3 N} \nu_{j}}{\prod_{j=1}^{3 N-1} \nu_{j}^{\ddagger}}
$$

where $\nu$ and $\nu^{\ddagger}$ are normal frequencies of vibration at the initial and saddle point states, respectively, and $N$ is the total number of atoms in the system. We find $\nu_{d}=50.1$, 1.5 , and $0.16 \mathrm{ps}^{-1}$ for Type I, II and III transitions, respectively. These numbers are comparable to the values found by other workers [61, 62].

\section{Hybrid continuum-KMC model}

In this section we describe the hybrid continuum-KMC method developed to model the heating of a nanobeam due to surface reconstruction.

\subsection{The continuum model}

In Section 4, we demonstrated that the continuum CV model in Eq. (5) provides a good description of temperature evolution in silicon nanobeams. However, a stochastic source term $r(x, t)$ must be added to the energy equation in Eq. (2) to model the latent heat released per unit time during the dimerization process: ${ }^{5}$

$$
\frac{\partial e}{\partial t}=-\frac{\partial q}{\partial x}+r
$$

\footnotetext{
5 See for example Section 10.2 in [9].
} 
Using the constitutive CV model for the heat flux in Eq. (4), the energy equation in Eq. (31) and the thermal conductivity $k$ in Eq. (9), yields the $\mathrm{CV}$ wave equation with a source term:

$$
\frac{\partial \theta}{\partial t}=-\tau_{q} \frac{\partial^{2} \theta}{\partial t^{2}}+\frac{k_{0}}{\gamma}\left[\left(\frac{\theta}{\theta_{0}}\right)^{s}\left(\frac{s}{\theta}\left(\frac{\partial \theta}{\partial x}\right)^{2}+\frac{\partial^{2} \theta}{\partial x^{2}}\right)\right]+r / \gamma
$$

To simplify the model, we assume that the thermal conductivity $k$ becomes constant for temperatures exceeding the boundary values: ${ }^{6}$

$$
k(\theta)= \begin{cases}k_{0}\left(\theta / \theta_{0}\right)^{s}, & \text { if } \theta \leq \theta_{L}, \\ k\left(\theta=\theta_{L}\right), & \text { otherwise, }\end{cases}
$$

where $k_{0}$ is the thermal conductivity at initial temperature $\theta_{0}, s$ is a real exponent and $\theta_{L}$ is the boundary temperature. We choose the value of $\tau_{q}$ obtained from the TPI-IRLS method described in Section 4.

We employ the explicit 1D finite difference discretization schemes described in the SI to numerically solve the PDE in Eq. (32). The spatial grid size is taken equal to the bin size in the NEMD simulations, so that the continuum grid points along the $x$-axis are located at $x_{n}(n=$ $1, \ldots, N_{\mathrm{b}}$ ) (the geometric centers of the NEMD bins) as defined in Section 3. The discretization time step is taken to be $\Delta t=0.001 \mathrm{ps}$ to ensure that the explicit scheme satisfies the Courant-Friedrichs-Lewy condition [64] for numerical stability. ${ }^{7}$

The heat source term $r=r(x, t)$ appearing in Eq. (32) accounts for the latent heat that is released whenever a dimer is formed. A dimerization event at position $x_{n}(n=$ $\left.2, \ldots, N_{\mathrm{b}}-1\right)$ and time $t$ is modeled as a uniform heat pulse of duration $\Delta t_{R}$. Thus over the time interval $\left[t, t+\Delta t_{R}\right]$, we have

$$
r\left(x_{n}, t\right)=\dot{Q}_{e},
$$

where $\dot{Q}_{e}$ is the energy released per unit volume per unit time. Since the volume associated with a grid point is $V_{\text {bin }}$ (volume of a bin) and a dimerization event releases $\Delta E_{r}$ energy, we have

$$
\dot{Q}_{e}=\frac{1}{V_{\mathrm{bin}}} \frac{\Delta E_{r}}{\Delta t_{R}} .
$$

\footnotetext{
${ }^{6}$ Adopting a temperature-dependent thermal conductivity as in Eq. (9) beyond the boundary temperatures requires the thermal parameter extraction process described in Section 3 to be done for temperatures exceeding $30 \mathrm{~K}$. This means taking into account the surface reconstruction process and would require a significant modification to the TPI-IRLS method. Moreover, it becomes difficult to handle the PDE with temperature dependent thermal conductivity due to the instabilities introduced by the presence of the stochastic term. In addition, the highly nonequilibrium nature of latent heat release leads to large sudden changes in the temperature gradients. This suggest the need for a nonlocal dependence of thermal conductivity on the temperature gradient field $[40,63]$. For these reasons, we have opted to use a simple constant thermal conductivity in this analysis for temperatures above the boundary temperature, reserving more complex models for future work.

${ }^{7}$ The time step violating the CFL condition for all cases in the present work is greater than $0.1 \mathrm{ps}$.
}

The energy $\Delta E_{r}$ for the dimerization types being considered is given in Section 5 .

We estimate the time interval $\Delta t_{R}$ over which the latent heat energy is absorbed in a bin based on the following heuristic argument. Consider a constant heat source $\dot{Q}_{e}$ located at the origin of an infinite medium. Assuming Fourier's model, the axisymmetric temperature field at a radial distance $\xi$ is given by [65]

$$
\theta(\xi, t)=\frac{\dot{Q}_{e}}{4 \pi k \xi} \operatorname{erfc}\left(\frac{\xi}{\sqrt{4 \alpha t}}\right),
$$

where $\alpha=k / \gamma$ is the thermal diffusivity and erfc is the error function. We identify $\Delta t_{R}$ with the time at which the temperature reaches $90 \%$ of its steady-state value at $\xi=\Delta_{x} / 2$ corresponding to the distance from a heat source at a bin center to its edges, i.e.

$$
\begin{array}{r}
\theta\left(\Delta_{x} / 2, \Delta t_{R}\right)=0.9 \lim _{t \rightarrow \infty} \theta\left(\Delta_{x} / 2, t\right), \\
\Longrightarrow \operatorname{erfc}\left(\frac{\Delta_{x} / 2}{\sqrt{4 \alpha t_{R}}}\right)=0.9 .
\end{array}
$$

The interval $\Delta t_{R}$ is an estimate for the time that a localized heat pulse generated by a dimerization event is uniformly distributed across the enclosing bin. For a nanobeam of size $33 \times 3 \times 3$ unit cells, $\Delta_{x}=3 a_{0}=16.29 \AA$. The thermal diffusivity for a nanobeam of this size follows from the results of Section 4 as $\alpha=k / \gamma=157.18 \AA^{2}$ ps, where $k=k_{0}\left(\theta / \theta_{0}\right)^{s}=28.43(30 / 10)^{-1.9}=3.53 \mathrm{~W} / \mathrm{mK}$ and $\gamma=1.40 \times 10^{-5} \mathrm{eV} /\left(\AA^{3} \mathrm{~K}\right)$. Using these values in Eq. (37), we obtain $\Delta t_{R}=0.08$ ps. The value of $\Delta t_{R}$ depends on the nanobeam size because thermal conductivity is size dependent. For systems of size $63 \times 3 \times 3$ and $93 \times 3 \times 3$, we find $\Delta t_{R}=0.06$ and $0.05 \mathrm{ps}$, respectively.

The numerical formulation for solving the PDE is now complete except for the determination of the dimerization events. This is a stochastic process that we model using the KMC algorithm described in the next section.

\subsection{The KMC method and the algorithm}

The source term $r(x, t)$ in Eq. (32) represents dimerization events on the surfaces of a nanobeam that inject heat pulses into the system. Since dimerization is a random activated process, we use a stochastic KMC algorithm to determine the locations and waiting times for dimerization events.

In the KMC method, a catalog (rate table) of all possible events is prepared at a given time. In our case, the events correspond to the formation of dimers at grid points associated with internal bin centers $\left(n=2, \ldots, N_{\mathrm{b}}-1\right)$. We make several assumptions to simplify the formulation and make it computationally tractable:

1. Each grid point is associated with a bin in the original NEMD simulation and therefore has a finite number of possible dimerizations $d_{\max }$, which depends on the top and bottom bin surface area. In the simulations reported below the bin surfaces are $3 \times 3$ unit 
cells, for which $d_{\max }=12$ on average. Once all 12 dimers are formed, further dimerization is not possible in that bin.

2. The first dimer on the top (001) and bottom (001) surfaces of each bin has to be of Type I as described in Section 5. Once a type I dimer forms, a maximum of five dimers of either Type II or Type III can form on that surface. However, to simplify the formulation we require two Type I dimers to form first, and then ten dimers of either Type II or Type III can be formed.

3. Only one grid point can undergo dimerization at a time. This greatly reduces the computational intensity of the KMC computations. This is a reasonable approximation since dimerization events are independent and the probability of two or more dimers forming simultaneously is negligible in comparison to single dimer formation. This is an important assumption since it reduces the number of events to $O\left(N_{\mathrm{b}}\right)$ making the calculation tractable.

A continuum-KMC formulation consistent with the above assumptions is given in Algorithm 1. Between dimerization events, the PDE in Eq. (32) is integrated without a source term. When a dimerization event occurs the PDE is integrated for a period $\Delta t_{R}$ (computed in the previous section) with the constant source term $\dot{Q}_{e}$ given in Eq. (35). The dimerization events and the times they occur are determined using a KMC algorithm for time-dependent transition rates due to Prados et al.[7]. (A time-dependent algorithm must be used because the temperature along the nanobeam is changing during the $\mathrm{KMC}$ process and hence the rates are changing.) The average waiting time $\Delta t_{\mathrm{KMC}}$ between events is obtained by solving

$$
\int_{0}^{\Delta t_{\mathrm{KMC}}} R_{\mathrm{tot}}\left(t^{\prime}\right) d t^{\prime}=-\ln u,
$$

where $u$ is a uniform random number in the range $[0,1]$, $R_{\text {tot }}(t)=\sum_{i=1}^{N_{p}} R_{i}(t)$ is the total transition rate at time $t, N_{p}$ is the number of events (all possible dimerization events at all grid points that can occur during the time interval $\left.\Delta t_{\mathrm{KMC}}\right)$, and $R_{i}\left(i=1, \ldots, N_{p}\right)$ are the rates for forming the dimers. We assume that at each grid point, two dimers of Type I form first (one on the top surface and one on the bottom) followed by ten dimers of either Type II or III. ${ }^{8}$ Thus the number of dimers $d_{n}$ at grid point $n$ ranges from 0 to 12 . The rate $R_{i}$ for an event

${ }^{8}$ This is clearly an approximation. A more accurate approach would track the topology of dimer formation on the surface and include all possible events. (For example Type II dimers should only form adjacent to Type I dimers, and more than two Type I dimers can in principle form.) However this would greatly increase the complexity of the code and its computational expense and it was felt that the simpler more approximate approach was consistent with the overall level of accuracy of the model. $\overline{\text { Algorithm } 1 \text { Continuum-KMC method for nanobeam }}$ temperature evolution in the presence of surface reconstructions.

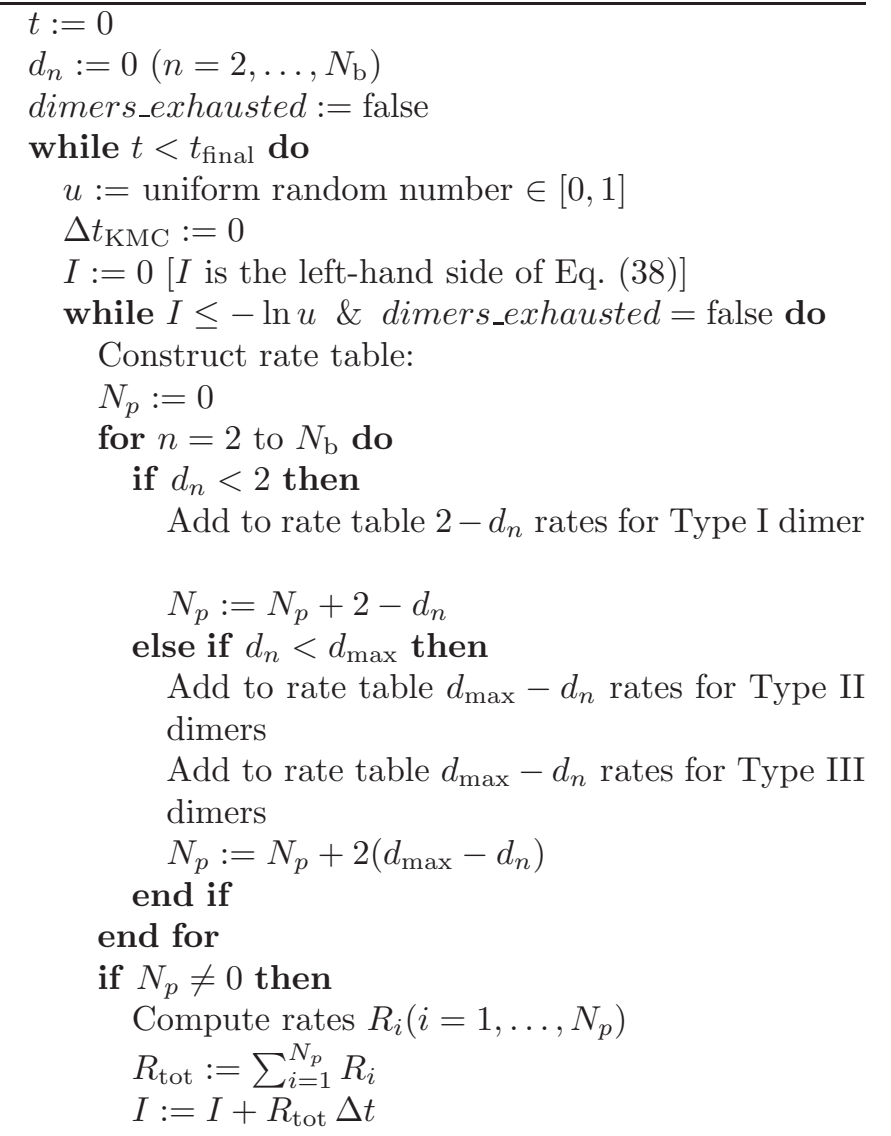
Integrate the PDE in Eq. (32) for one discretization time step with zero source term

$t:=t+\Delta t$

else

$\Delta t_{\mathrm{KMC}}:=\Delta t_{\mathrm{KMC}}+\Delta t$

dimers_exhausted $:=$ true

end if

end while

if dimers_exhausted $=$ false then

$\widetilde{R}_{i}:=\sum_{j=1}^{i} R_{j}$

$\mathrm{u}:=$ uniform random number $\in[0,1]$

Select event $i$ for which $\widetilde{R}_{i-1}<u \widetilde{R}_{N_{p}} \leq \widetilde{R}_{i}$

$d_{n}:=d_{n}+1$ [where $n$ is bin associated with event $i]$

Integrate the PDE in Eq. (32) for $\Delta t_{R}$ time with constant heat source $\dot{Q}_{e}$ given in Eq. (35)

$t:=t+\Delta t_{R}$

else

Integrate the PDE in Eq. (32) for one discretization time step with zero source term

$t:=t+\Delta t$

end if

end while 
occurring at grid point $x_{n}$ is computed from Eq. (29) with $\theta=\theta_{n}(t)$, and the attempt frequency $\nu_{d}$ and activation barrier $\Delta E_{b}$ for a Type I, Type II, Type III dimer given in Section 5. Once the time to the next event $\Delta t_{\mathrm{KMC}}$ has been obtained by solving Eq. (38), the rate table at that time is constructed and the event that happens is randomly selected in standard KMC fashion as described in Algorithm 1. Once all dimerization sites are exhausted, the KMC algorithm is no longer applied and the PDE in Eq. (32) is integrated without a source term from then on.

\subsection{Numerical results}

We now revisit the problem described in Section 1 and the results shown in Fig. 1. A silicon nanobeam with exposed $\{001\}$ surfaces is equilibrated at $10 \mathrm{~K}$ and then its ends are heated to $30 \mathrm{~K}$ and maintained at this temperature as a fixed thermal BC. As the temperature of the interior begins to rise, dimerization events begin to occur that due to the latent heat released cause the nanobeam temperature in the center to dramatically overshoot the $\mathrm{BC}$ temperature.

In this section, we simulated this problem using the continuum-KMC model outlined in Algorithm 1 using the thermal parameters for the CV model obtained using the TPI-IRLS method described in Section 4. Three different nanobeam sizes are studied: $33 \times 3 \times 3,63 \times 3 \times 3$, and $93 \times 3 \times 3$. The models contain 2574,4914 and 7254 atoms respectively. NEMD simulations were carried out on a parallel cluster using LAMMPS with 24 cores. Simulation times for the three systems were $2.2,4.5$ and 8 hours, respectively. The continuum-KMC calculations were carried out using the $\mathrm{CV}$ parameters given in Table 1. The results comparing the NEMD profiles (red) and continuum-KMC predictions (blue) are shown in Fig. 8. (Note that the results shown in Fig. 1, correspond to Fig. 8(c) here.) The plots show a series of temperature profiles at increasing time. The continuum-KMC results are in excellent agreement with the NEMD results, correctly capturing the thermal overshooting. Although not apparent in the figure, the temperature first rises to reach the maximum temperature profile and then cools down to the BC temperature of $30 \mathrm{~K}$ as heat is dissipated through the thermostated ends. The times it takes the nanobeams to reach the maximum temperature profile and then cool back down to the BC temperature are: $110 \mathrm{ps} / 300 \mathrm{ps}(33 \times 3 \times 3), 220 \mathrm{ps} / 600 \mathrm{ps}$ $(63 \times 3 \times 3)$, and $234 \mathrm{ps} / 900 \mathrm{ps}(93 \times 3 \times 3)$. These times may be sensitive to the damping parameter used in the thermostated bins at the ends of the nanobeam.

Having established the validity of the continuum-KMC model for smaller system sizes it is of interest to explore its predictions for larger systems that are difficult to simulate using NEMD due to the computational cost. Figure 9 shows the results for a nanobeam of length 603 unit cells $(0.327 \mu \mathrm{m})$. The $\mathrm{CV}$ parameters were estimated as $k_{0}=$ $303.13 \mathrm{~W} / \mathrm{mK}, \tau_{q}=6.11 \mathrm{ps}, s=-1.9$. The dimer latent heat absorption time is taken to be $\Delta t_{R}=0.0065 \mathrm{ps}$. The thermal overshooting in this case is even more significant.

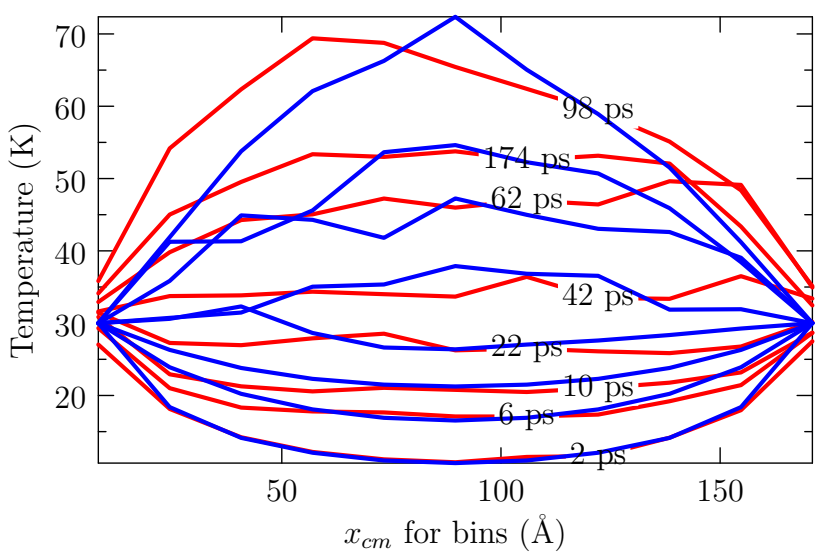

(a)

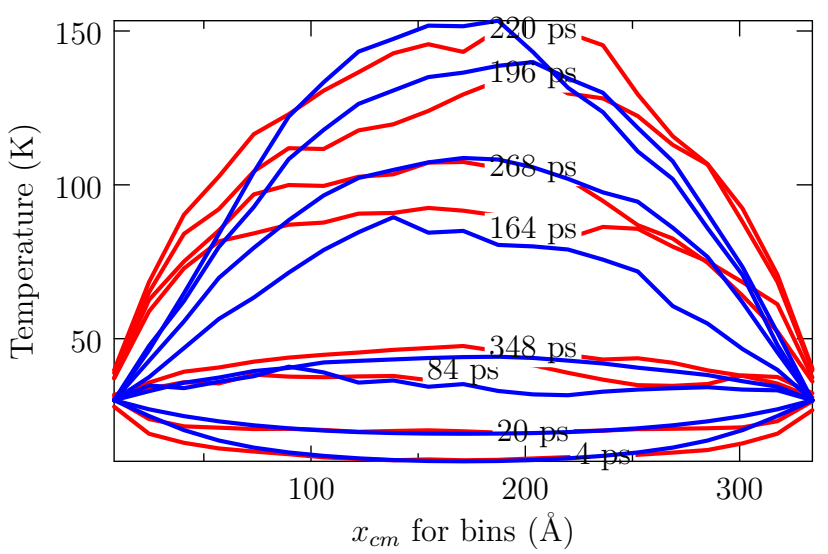

(b)

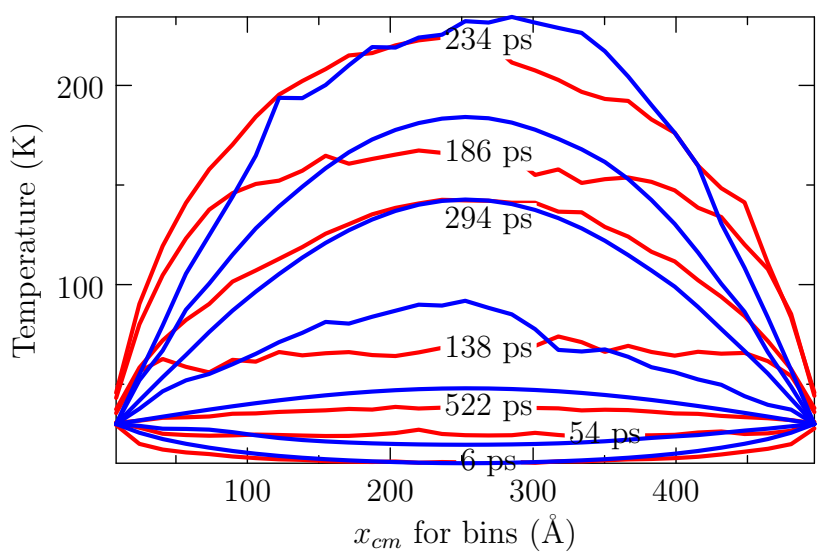

(c)

Figure 8: Comparison of the NEMD temperature profiles (red) with the predictions of a hybrid continuum-KMC CV model (blue) for silicon nanobeams of size (a) $33 \times 3 \times 3$, (b) $63 \times 3 \times 3$, and (c) $93 \times 3 \times 3$ unit cells with free (001) surfaces. The initial beam temperature is $\theta_{0}=10 \mathrm{~K}$ and the boundary temperatures are set to $30 \mathrm{~K}$. In all simulations, NEMD profiles are averaged over ten different initial momenta distribution. The hybrid continuum-KMC profiles are averaged over five simulations with different seeds for the pseudo random number generator used by the KMC calculations. Each profile is an average of 1 ps 2 ps and 3 ps of data for three different sizes, respectively. The profiles are marked with their corresponding times. For the sake of clarity many profiles have been omitted. 


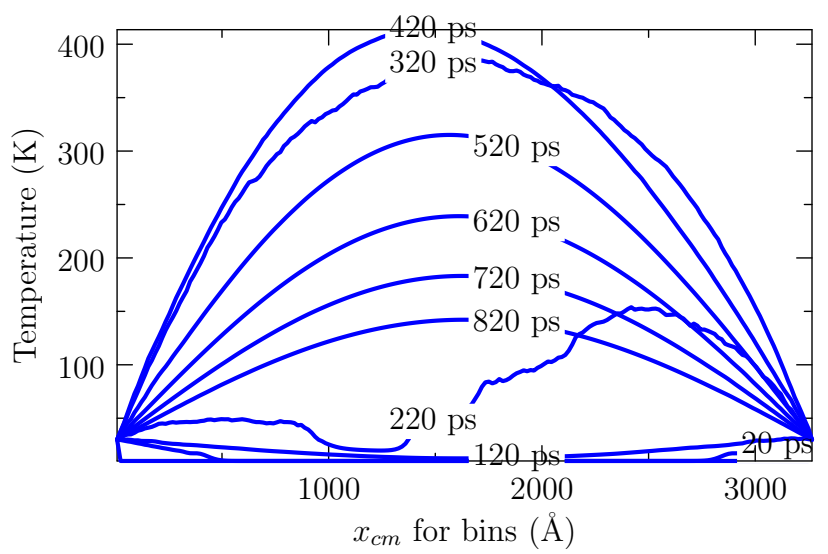

Figure 9: Hybrid continuum-KMC temperature profiles for a silicon nanobeam of size $603 \times 3 \times 3$ unit cells when the initial temperature $\theta_{0}=10 \mathrm{~K}$ and the boundary temperatures were raised to $\theta_{L}=\theta_{R}=$ $30 \mathrm{~K}$. The thermal parameters are $k_{0}=303.13 \mathrm{~W} / \mathrm{mK}, \tau_{q}=6.11 \mathrm{ps}$, $s=-1.9$, and $\Delta t_{R}=0.0065 \mathrm{ps}$. The profiles are marked with their corresponding times. For the sake of clarity many profiles have been omitted.

In general, we find that the degree of superheating, characterized by the maximum temperature along the beam,

$$
\theta_{\max }=\max \{\theta(x, t) \mid 0 \leq x \leq L, 0 \leq t<\infty\},
$$

increases with increasing nanobeam length $L$ as shown in Fig. 10(a). The same data is plotted on a semi-log plot in Fig. 10(b). A linear fit of the form,

$$
\theta_{\max }(L)=a \ln L-b,
$$

where $a=75.91 \pm 3.79 \mathrm{~K} / \ln \mathrm{nm}, b=1.93 \pm 1.07 \mathrm{~K}$ with $\theta_{\max }$ in $\mathrm{K}$ and $L$ in nm provides a good fit to the data above a length of 123 unit cells $(L=123 \times 0.543=66.8 \mathrm{~nm})$. The deviation from the logarithmic fit can be understood by considering the length-dependence of the thermal conductivity and time lag shown in Figs. 11(a) and 11(b). Above about 123 unit cells $\left(1 / L=0.015 \mathrm{~nm}^{-1}\right)$, the dependence of thermal conductivity $k_{0}$ on length $L$ takes the following form $[40,41]$ :

$$
\frac{1}{k_{0}}=\frac{1}{k_{\infty}}+\frac{c}{L}
$$

where $c$ is a constant and $k_{\infty}$ is the bulk value at $10 \mathrm{~K}$. Below 123 unit cells the fit breaks down, which explains the deviation seen in Fig. 10(b). Fitting the data in in Fig. 11(a) to the relation in Eq. (41), yields $k_{\infty}=2676.70 \pm$ $124.84 \mathrm{~W} / \mathrm{mK}$, which is close to the experimental result of Thompson and Younglove [66]. The dependence of $\tau_{q}$ on $L$ takes a similar form $[17,41]$ :

$$
\frac{1}{\tau_{q}}=\frac{1}{\tau_{\infty}}+\frac{c_{\tau}}{L}
$$

where $c_{\tau}$ is a constant dependent on the thermostat parameters [40]. Fitting to the data in Fig. 11(b), we find that the bulk value $\tau_{\infty}$ is $8.67 \pm 0.37 \mathrm{ps}$.

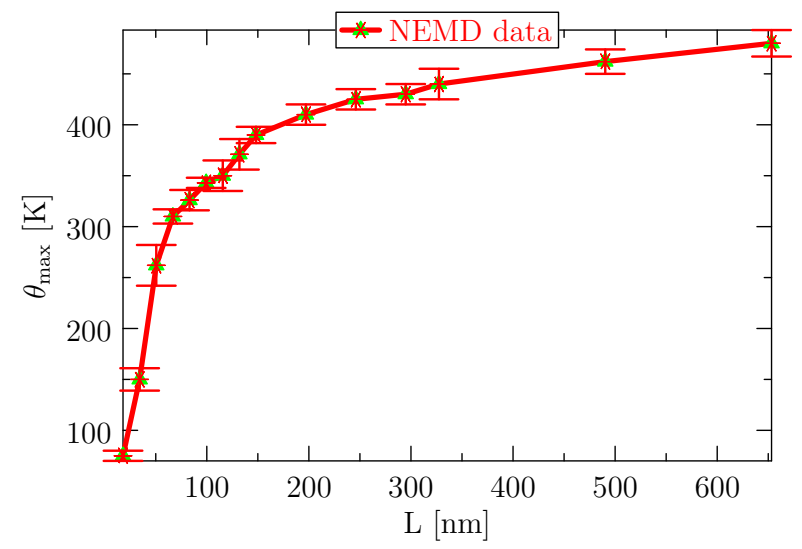

(a)

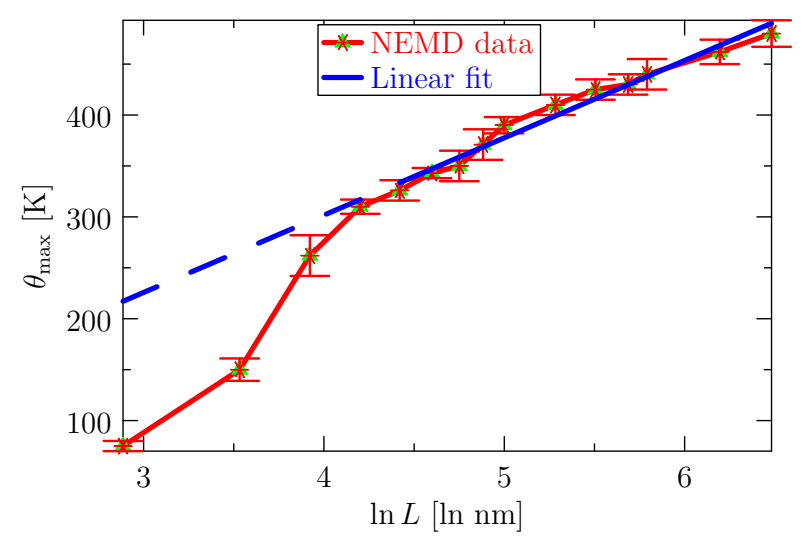

(b)

Figure 10: (a) Plot of $\theta_{\max }$ versus $L$. The maximum value for a given length is obtained by averaging over ten different ensembles. The error bars are the standard deviations in the bin temperature over these ensembles. (b) Plot of $\theta_{\max }$ versus the natural $\log$ of $L$. The data for lengths smaller than 123 unit cells $(\ln L=4.2$, where $L=123 \times 0.543=66.8 \mathrm{~nm}$ ) were not included in the curve fitting. The dashed line shows the extension of the fit to smaller lengths.

The increase of the superheating temperature $\theta_{\max }$ with nanobeam length seen in Fig. 10 can be understood from an analysis of the spatially non-dimensionalized form of Eq. (32),

$\frac{\partial \theta}{\partial t}=-\tau_{q} \frac{\partial^{2} \theta}{\partial t^{2}}+\frac{k_{0}(L)}{\gamma L^{2}}\left[\left(\frac{\theta}{\theta_{0}}\right)^{s}\left(\frac{s}{\theta}\left(\frac{\partial \theta}{\partial x^{\prime}}\right)^{2}+\frac{\partial^{2} \theta}{\partial x^{\prime 2}}\right)\right]+r^{\prime} / \gamma$

where $x^{\prime}=x / L$, and $r^{\prime}\left(x^{\prime}, t\right)$ is the dimensionless source term. As the length of the nanobeam increases, the diffusion term coefficient $k_{0}(L) / \gamma L^{2}$ tends to zero. The wave term does not play an important role due to the small value of $\tau_{q}$ in this system. As a result heat is transported at a slower rate as the nanobeam length increases. At the same time, the source term $r^{\prime}\left(x^{\prime}, t\right)$ tends to infinity as the number density of surface dimerization sites increases over the unit length. Basically as the length of the nanobeam increases more heat is generated and the ability of the nanobeam to diffuse the heat away decreases. This trend will continue until the maximum temperature reaches the 


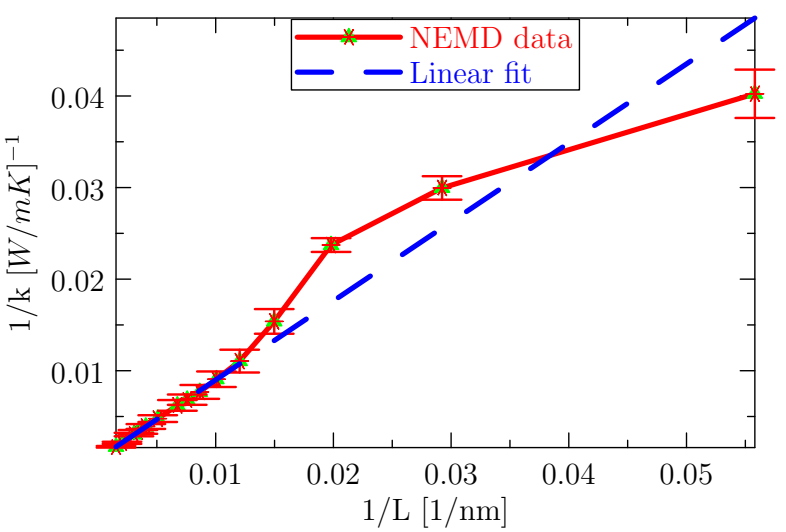

(a)

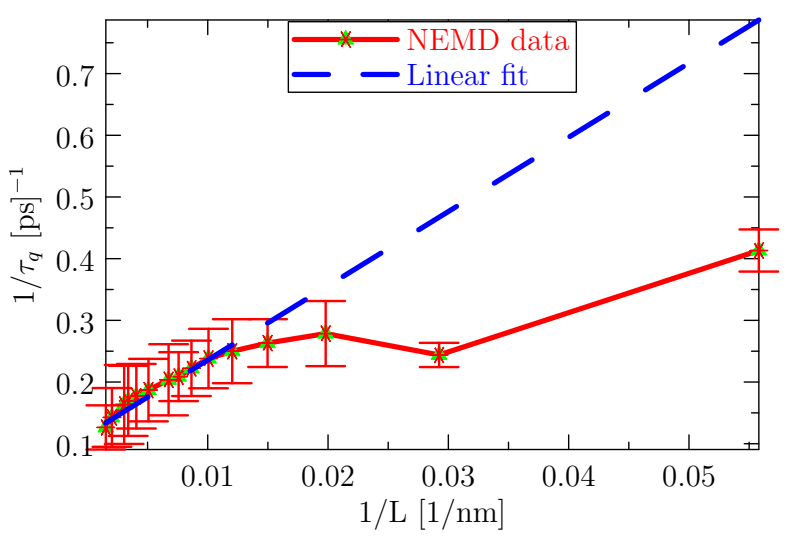

(b)

Figure 11: Plots of (a) $1 / k_{0}$ versus $1 / L$, (b) $1 / \tau_{q}$ versus $1 / L$, where data for lengths smaller than 123 unit cells $\left(1 / L>0.015 \mathrm{~nm}^{-1}\right)$ are not included in the linear fit.

melting point. From Eq. (40) and the melting point of Si $\left(\theta_{\mathrm{m}} \approx 1700 \mathrm{~K}\right)$, the length required for this to happen is about $5.45 \mathrm{~m}$.

\section{Conclusion}

Nanomaterials can undergo large temperature changes due to the release of latent heat accompanying surface reconstruction and phase transformations. An example observed in NEMD simulations is the heating of silicon nanobeams due to the reconstruction of $\{001\}$ to form dimers along $\langle 110\rangle$ directions. For example NEMD simulations using the SW potential show that a silicon nanobeam of size $505 \times 16.3 \times 16.3 \AA$ that is initially at $10 \mathrm{~K}$ and then heated at the ends to $30 \mathrm{~K}$, exhibits superheating reaching above $200 \mathrm{~K}$ at its center. The maximum temperature diverges logarithmically with beam length until the melting temperature is reached.

We use the silicon nanobeam problem as a motivating example to develop a general hybrid continuum-KMC methodology for simulating superheating behavior. In this approach, thermal transport is modeled using a non-Fourier continuum model in which heat propagates as a wave with a finite velocity. In the first part of the paper, non-Fourier thermal parameters are obtained from NEMD simulations with the SW potential using a novel TPI-IRLS regression method. Results for the non-Fourier CV and Jeffreystype models, as well as for Fourier's model, for different nanobeam sizes are given in Section 4. Based on these results, it is determined that the CV model is best able to capture thermal transport observed in NEMD simulations of silicon nanobeams.

In the second part of the paper, the $\mathrm{CV}$ wave equation is augmented with a source term to account for the energy injected into the system by the latent heat released during dimer formation accompanying surface reconstruction. The dimerization process is stochastic and is modeled using a KMC algorithm for time-dependent transition rates that is coupled to the continuum $\mathrm{CV}$ equation. The rates needed for the KMC algorithm are estimated using hTST with activation barriers obtained using CI-NEB and attempt frequencies computed using Vineyard's equation. The resulting continuum-KMC method is described in Algorithm 1. The predictions of the method are found to be in excellent agreement with the NEMD simulation results.

Although the silicon nanobeam example is academic in nature (since surface reconstruction in silicon is observed experimentally at temperatures as low as $5 \mathrm{~K}$ under vacuum conditions), the simulation methodology developed here can be applied to range of similar problems in which surface reconstructions or phase transformations occur at higher temperatures under ambient conditions $[1,67,68]$. This is a promising area for future research.

\section{Acknowledgment}

This research was partly supported through the National Science Foundation (NSF) under grants PHY-0941493 and DMR-1408211, and the Air Force Office of Scientific Research (AFOSR) under Grant No. FA9550-09-1-0157.

\section{References}

[1] H. Zheng, J. B. Rivest, T. A. Miller, B. Sadtler, A. Lindenberg, M. F. Toney, L. Wang, C. Kisielowski, A. P. Alivisatos, Observation of transient structural-transformation dynamics in a $c u_{2} s$ nanorod, Science 333 (6039) (2011) 206-209.

[2] M.-C. Desjonquères, D. Spanjaard, Concept in surface physics, Springer-Verlag, Heidelberg, Berlin, Germany, 1993.

[3] P. Perdigão, D. Deresmes, B. randidier, M. Dubois, C. Delerue, G. Allan, D. Stiévenard, Semiconducting surface reconstructions of p-type $\mathrm{Si}(100)$ substrates at 5 k, Phys. Rev. Lett. 92 (21) (2004) 216101-1-216101-4.

[4] D. D. Joseph, L. Preziosi, Heat Waves, Rev. Mod. Phys. 61 (1) (1989) 41-73.

[5] A. Guitton, D. J. Verschuur, Adaptive subtraction of multiples using the L1-norm, Geophys. Prospect. 52 (1) (2004) 27-38.

[6] A. Chatterjee, D. G. Vlachos, An overview of spatial microscopic and accelerated kinetic Monte Carlo methods, J. Comput.-Aided Mater. Des. 14 (2) (2007) 253-308.

[7] A. Prados, J. J. Brey, B. Sánchez-Rey, A dynamical Monte Carlo algorithm for master equations with time-dependent transition rates, J. Stat. Phys. 89 (1997) 709-734. 
[8] G. Henkelman, B. P. Uberuaga, H. Jónsson, A climbing image nudged elastic band method for finding saddle points and minimum energy paths, J. Chem. Phys. 113 (2000) 9901-9904.

[9] E. B. Tadmor, R. E. Miller, R. S. Elliott, Continuum Mechanics and Thermodynamics, Cambridge University Press, Cambridge, 2012.

[10] C. Cattaneo, Sulla conduzione del calore, Atti. Sem. Mat. Fis. Univ. Modena 3 (1948) 83-101.

[11] C. Cattaneo, Sur une forme de l'equation de la chaleur eliminant le paradoxe d'une propagation instantanée, C. R. Acad. Sci. Paris 247 (1958) 431-433.

[12] P. Vernotte, Les paradoxes de la theorie continue de l'equatioin de la chaleur, C. R. Acad. Sci. 246 (1958) 3154-3155.

[13] P. Vernotte, La véritable équation de la chaleu, C. R. Acad. Sci. 247 (1958) 2103-2105.

[14] M. E. Gurtin, A. C. Pipkin, A general theory of heat conduction with finite wave speeds, Arch. Rational Mech. Anal. 31 (22) (1968) 113-126.

[15] J. W. Nunziato, On heat conduction in materials with memory, Quart. Appl. Math. 29 (2) (1971) 187-204.

[16] H. Jeffreys, The Earth: Its Origin, History and Physical Constitution, 8th Edition, Cambridge University Press, Cambridge, 1976.

[17] A. Singh, E. B. Tadmor, Thermal parameter identification for non-Fourier heat transfer from molecular dynamics, J. Comp. Phys. 299 (2015) 667-686.

[18] T. G. Müller, J. Timmer, Parameter identification techniques for partial differential equations, Int. J. Bifurcat. Chaos, 14 (6) (2004) 2053-2060.

[19] I. J. Schoenberg, Spline functions and the problem of graduation, Proc. Nat. Acad. Sci. USA 52 (1964) 947-950.

[20] C. H. Reinsch, Smoothing by spline functions, Numer. Math. 10 (1967) 177-183.

[21] C. d. Boor, A Practical Guide to Splines, revised Edition, Springer Verlag, New York, 2001.

[22] O. M. Alifanov, Inverse Heat Transfer Problems, Springer Verlag, Berlin Heidelberg, 1994.

[23] F. H. Stillinger, T. A. Weber, Computer simulation of local order in condensed phases of silicon, Phys. Rev. B 31 (8) (1985) $5262-5271$.

[24] A. Singh, A three-body Stillinger-Weber (SW) model (parameterization) for Silicon, https://openkim.org/cite/MO_405512056662_001 (2013).

[25] A. Singh, A three-body Stillinger-Weber (SW) potential for Silicon, https://openkim.org/cite/MD_335816936951_001 (2013).

[26] E. B. Tadmor, R. S. Elliott, J. P. Sethna, R. E. Miller, C. A. Becker, The potential of atomistic simulations and the Knowledgebase of Interatomic Models, JOM 63 (2011) 17.

[27] E. B. Tadmor, R. E. Miller, Modeling Materials, Cambridge University Press, Cambridge, 2011.

[28] S. Nosé, A unified formulation of the constant temperature molecular dynamics methods, J. Chem. Phys. 81 (1) (1984) 511519 .

[29] W. G. Hoover, Canonical dynamics: Equilibrium phase-space distributions, Phys. Rev. A 31 (3) (1985) 1695-1697.

[30] G. J. Martyna, M. L. Klein, M. Tuckerman, Nosé-hoover chains: The canonical ensemble via continuous dynamics, J. Chem. Phys. 97 (4) (1992) 2635-2645.

[31] D. Frenkel, B. Smit, Understanding Molecular Simulation, 2nd Edition, Academic Press, San Diego, CA, US, 2002.

[32] T. Schneider, E. Stoll, Molecular-dynamics study of a threedimensional one-component model for distortive phase transitions, Phys. Rev. B 17 (3) (1978) 1302-1322.

[33] D. J. Evans, G. Morriss, Statistical Mechanics of Nonequilibrium Liquids, 2nd Edition, Cambridge University Press, New York, US, 2008.

[34] J. Jellinek, D. H. Li, Separation of the energy of overall rotation in any N-body system, Phys. Rev. Lett. 62 (3) (1989) 241-244.

[35] S. J. Plimpton, Fast parallel algorithms for short-range molecular dynamics, J. Comput. Phys. 117 (1995) 1-19.

[36] LAMMPS website, http://lammps.sandia.gov/.
[37] W. Shinoda, M. Shiga, M. Mikami, Rapid estimation of elastic constants by molecular dynamics simulation under constant stress, Phys. Rev. B 69 (13) (2004) 134103-1-134103-8.

[38] M. P. Allen, D. J. Tildesley, Computer Simulation of Liquids, Oxford University Press, New York, 1987.

[39] L. Ott, M. Longnecker, An introduction to statistical methods and data analysis, 1st Edition, Duxbury Press, California, 2001.

[40] A. Singh, E. B. Tadmor, Removing artificial kapitza effects from bulk thermal conductivity calculations in direct molecular dynamics, J. Appl. Phys. 117 (2015) 185101.

[41] D. P. Sellan, E. S. Landry, J. E. Turney, A. J. H. McGaughey, C. H. Amon, Size effects in molecular dynamics thermal conductivity predictions, Phys. Rev. B 81 (2010) 214305.

[42] P. K. Schelling, S. R. Phillpot, P. Keblinski, Comparison of atomic-level simulation methods for computing thermal conductivity, Phys. Rev. B 65 (14) (2002) 144306.

[43] J. W. Roh, S. Y. Jang, J. Kang, S. Lee, J.-S. Noh, W. Kim, J. Park, W. Lee, Size-dependent thermal conductivity of individual single-crystalline PbTe nanowires, Appl. Phys. Lett. 96 (2010) 103101.

[44] R. E. Schlier, H. Farnsworth, Structure and adsorption characteristics of clean surfaces of Germanium and Silicon, J. Chem. Phys. 30 (1959) 917.

[45] J. J. Lander, J. Morrison, Low voltage electron diffraction study of the oxidation and reduction of silicon, J. Appl. Phys. 33 (6) (1962) 2089-2092.

[46] T. D. Poppendieck, T. C. Ngoc, M. B. Webb, An electron diffraction study of the structure of silicon (100), Surf. Sci. 75 (2) (1978) 287-315.

[47] R. M. Tromp, R. J. Hamers, J. E. Demuth, Dimer structure observed with scanning tunneling microscopy, Phys. Rev. Lett. 55 (12) (1985) 1303-1306.

48] Y. Kondo, T. Amakusa, M. Iwatsuki, H. Tokumoto, Phase transition of the $\mathrm{Si}(001)$ surface below 100 k, Surf. Sci. 453 (2000) L318-L322.

[49] Y. J. Li, Y. Sugawara, C. Hobbs, Y. Naitoh, M. Kageshima, H. Nomura, N. Ozaki, L. Kantorovich, Origin of $\mathrm{p}(2 \mathrm{x} 1)$ phase on $\mathrm{Si}(001)$ by noncontact atomic force microscopy at $5 \mathrm{~K}$, Phys. Rev. Lett. 96.

50] G. Le Lay, A. Cricenti, C. Ottaviani, P. Perfetti, T. Tanikawa, I. Matsuda, S. Hasegawa, Evidence of asymmetric dimers down to $40 \mathrm{k}$ at the clean $\mathrm{Si}(100)$ surface, Phys. Rev. B 66 (15) (2002) 153317-1-153317-4.

[51] M. Ono, A. Kamoshida, N. Matsuura, E. Ishikawa, T. Eguchi, Y. Hasegawa, Dimer buckling of the $\operatorname{Si}(001) 2 \times 1$ surface below $10 \mathrm{k}$ observed by low-temperature scanning tunneling microscopy, Phys. Rev. B 67 (20) (2003) 201306-1-201306-4.

[52] C. Guo, K. Hermann, Y. Zhao, Dynamics and energetics of reconstruction at the $\mathrm{Si}(100)$ surface, J. Phys. Chem. C 118 (2014) 25614-25619.

[53] M. T. Yin, M. L. Cohen, Theoretical determination of surface atomic geometry: Si(001)-(2x1), Phys. Rev. B 24 (4) (1981) 2303-2306.

[54] I. P. Batra, Atomic structure of the $\mathrm{Si}(001)-(2 x 1)$ surface, Phys. Rev. B 41 (8) (1990) 5048-5054.

[55] N. Roberts, R. J. Needs, Total energy calculations of dimer reconstructions on the silicon (001) surface, Surf. Sci. 236 (1990) $112-121$.

[56] J. Dabrowski, M. Scheffler, Self-consistent study of the electronic and structural properties of the clean $\operatorname{Si}(001)(2 \times 1)$ surface, Appl. Surf. Sci. 56 (1992) 15-19.

[57] R. A., G. Brocks, P. J. Kelly, Theoretical study of the $\mathrm{Si}(100)$ surface reconstruction, Phys. Rev. B 51 (20) (1995) 1450414524.

[58] E. T. Gawlinski, J. D. Gunton, Molecular-dynamics simulation of molecular-beam epitaxial growth of the silicon (100) surface, Phys. Rev. B 36 (9) (1987) 4774-4781.

[59] J. Lampinen, R. M. Nieminen, K. Kaski, Molecular dynamics simulation of epitaxial growth of the si(001) surface, Surf. Sci. 203 (1-2) (1988) 201-211.

[60] G. H. Vineyard, Frequency factors and isotope effects in solid 
state rate processes, J. Phys. Chem. Solids 3 (1-2) (1957) 121127.

61] G. Henkelman, H. Jónsson, Long time scale kinetic Monte Carlo simulations without lattice approximation and predefined event table, J. Chem. Phys. 115 (21) (2001) 9657-9666.

[62] T. Lazauskas, S. D. Kenny, R. Smith, Influence of the prefactor to defect motion in $\alpha$-iron during long time scale simulations, J. Phys. Condens. Matter 26 (39) (2014) 395007.

[63] P. B. Allen, Size effects in thermal conduction by phonons, Phys. Rev. B. 90 (2014) 054301

[64] R. Courant, K. Friedrichs, H. Lewy, Über die partiellen differenzengleichungen der mathematischen physik, Mathematische Annalen 100 (1) (1928) 32-74.

[65] H. D. Baehr, K. Stephan, Heat and mass transfer, 3rd Edition, Springer, San Diego, CA, US, 2011.

[66] J. C. Thompson, B. A. Younglove, Thermal conductivity of silicon at low temperatures, J. Phys. Chem. Solids 20 (1961) 146149 .

[67] T. Q. Qiu, C. L. Tien., Short-pulse laser heating on metals, Int. J. Heat Mass Transfer 35 (3) (1992) 719-726.

68] P. Galenko, D. Jou, Diffuse-interface model for rapid phase transformations in nonequilibrium systems, Phys. Rev. E 71 (2005) 046125. 